\title{
Blocking the Thrombin Receptor Promotes Repair of Demyelinated Lesions in the Adult Brain
}

\author{
Hyesook Yoon, ${ }^{1}$ Chan-Il Choi, ${ }^{1}$ Erin M. Triplet, ${ }^{2}$ Monica R. Langley, ${ }^{1}$ Laurel S. Kleppe, ${ }^{1}$ Ha Neui Kim, ${ }^{1}$ \\ Whitney L. Simon, ${ }^{1}$ and ${ }^{-I s o b e l ~ A . ~ S c a r i s b r i c k ~}{ }^{1,2,3}$ \\ ${ }^{1}$ Department of Physical Medicine and Rehabilitation, Rehabilitation Medicine Research Center, ${ }^{2}$ Neuroscience Program, and ${ }^{3}$ Department of Physiology \\ and Biomedical Engineering, Mayo Clinic School of Biomedical Sciences, Rochester, Minnesota 55905
}

Myelin loss limits neurological recovery and myelin regeneration and is critical for restoration of function. We recently discovered that global knock-out of the thrombin receptor, also known as Protease Activated Receptor 1 (PAR1), accelerates myelin development. Here we demonstrate that knocking out PAR1 also promotes myelin regeneration. Outcomes in two unique models of myelin injury and repair, that is lysolecithin or cuprizone-mediated demyelination, showed that PAR1 knock-out in male mice improves replenishment of myelinating cells and remyelinated nerve fibers and slows early axon damage. Improvements in myelin regeneration in PAR1 knock-out mice occurred in tandem with a skewing of reactive astrocyte signatures toward a prorepair phenotype. In cell culture, the promyelinating effects of PAR1 loss of function are consistent with possible direct effects on the myelinating potential of oligodendrocyte progenitor cells (OPCs), in addition to OPC-indirect effects involving enhanced astrocyte expression of promyelinating factors, such as BDNF. These findings highlight previously unrecognized roles of PAR1 in myelin regeneration, including integrated actions across the oligodendrocyte and astroglial compartments that are at least partially mechanistically linked to the powerful BDNF-TrkB neurotrophic signaling system. Altogether, findings suggest PAR1 may be a therapeutically tractable target for demyelinating disorders of the CNS.

Key words: astrocyte; brain derived neurotrophic factor; myelin; oligodendrocyte progenitor cells; protease activated receptor; regeneration

\section{Significance Statement}

Replacement of oligodendroglia and myelin regeneration holds tremendous potential to improve function across neurological conditions. Here we demonstrate Protease Activated Receptor 1 (PAR1) is an important regulator of the capacity for myelin regeneration across two experimental murine models of myelin injury. PAR1 is a G-protein-coupled receptor densely expressed in the CNS, however there is limited information regarding its physiological roles in health and disease. Using a combination of PAR1 knock-out mice, oligodendrocyte monocultures and oligodendrocyte-astrocyte cocultures, we demonstrate blocking PAR1 improves myelin production by a mechanism related to effects across glial compartments and linked in part to regulatory actions toward growth factors such as BDNF. These findings set the stage for development of new clinically relevant myelin regeneration strategies.

\section{Introduction}

Myelin dysfunction is recognized as a prominent feature of diverse neurological conditions affecting the adult and developing

Received Aug. 19, 2019; revised Dec. 15, 2019; accepted Dec. 17, 2019.

Author contributions: H.Y., C.-I.C., E.M.T., W.L.S., and I.A.S. designed research; H.Y., C.-I.C., E.M.T., M.R.L., L.S.K., H.N.K., W.L.S., and I.A.S. performed research; H.Y., C.-I.C., E.M.T., H.N.K., W.L.S., and I.A.S. analyzed data; H.Y., E.M.T., M.R.L., and W.L.S. edited the paper; I.A.S. wrote the first draft of the paper; I.A.S. wrote the paper.

This work was supported by the National Institutes of Health (Grant R01NS052741-10, R21NS107946, Pilot Project G-1510-06548), the National Multiple Sclerosis Society (Research Grants G-1508-05951 and RG-190133209), and by an Accelerated Regenerative Medicine Award from the Mayo Clinic Center for Regenerative Medicine (IAS) and the Eugene and Marcia Applebaum Fellowship from the Center for MS and Autoimmune Neurology (MRL).

The authors declare no competing financial interests.

Correspondence should be addressed to Isobel A. Scarisbrick at Scarisbrick.Isobel@mayo.edu.
CNS. Oligodendrocytes generate myelin across the neuraxis thereby enhancing nerve impulse conduction and provide critical trophic and metabolic axon support (Stassart et al., 2018). Oligodendroglia are vulnerable to injury in multiple sclerosis (MS) and other neuropathologies leading to conduction block, axonopathy and permanent neurologic impairment. Although the CNS has an innate capacity for myelin regeneration, this ability is impaired in CNS demyelinating diseases such as MS, leaving axons vulnerable to dysfunction and degeneration. 
There are at least 15 different drugs currently available to treat MS and the majority of these target inflammation, but do little to prevent progression (Bothwell, 2017). Ongoing axonal protection and long-term recovery of function will depend on new strategies to protect oligodendrocytes and their progenitors and to regenerate myelin sheaths. There is evidence to support an innate potential for myelin repair in the adult CNS and indeed axon remyelination is observed in some, but far from all MS lesions (Yeung et al., 2019). In some cases, oligodendrocyte progenitor cells (OPCs) are present in MS lesions even when remyelination is absent or incomplete (Smith et al., 1979; Patrikios et al., 2006). To improve remyelination in MS lesions, therefore, there is a crucial need to develop strategies to increase OPC availability and their ability to differentiate into mature myelinating oligodendrocytes.

Among the factors deregulated in demyelinating disorders, including MS, are a vast array of proteolytic enzymes (Scarisbrick, 2008). We previously showed that in excess serine proteases such thrombin or kallikrein 6 (Klk6) actively suppress oligodendrocyte differentiation, including process outgrowth and stability, and production of the major myelin proteins myelin basic protein (MBP) and proteolipid protein (PLP) (Scarisbrick et al., 2002; Burda et al., 2013). We discovered that these myelin suppressive effects are governed by their ability for cleavage-induced activation of a G-protein-coupled receptor referred to as the thrombin receptor or Protease Activated Receptor 1 (PAR1) (Burda et al., 2013; Choi et al., 2018). Through this mechanism, serine proteases signal in a hormone-like fashion, effectively communicating information to a cell about the extracellular microenvironment.

PAR1 plays critical roles in myelin development with PAR1 knock-out mice showing an accelerated pattern of myelin development and higher numbers of myelinating cells in the adult brain and spinal cord (Yoon et al., 2015; Choi et al., 2018). Moreover, PAR1 knock-out OPCs differentiate more readily in cell culture (Yoon et al., 2015). Based on these findings, we hypothesize that the PAR1 receptor is a molecular switch that when turned on suppresses myelination, including oligodendrocyte production and differentiation, both developmentally and in the context of myelin regeneration. We further hypothesize that this switch can be turned off to enhance the capacity for myelin regeneration. To test this hypothesis, we investigated the impact of PAR1 gene knock-out on myelin regeneration in two distinct murine models where demyelination is induced focally in the spinal cord by lysolecithin microinjection, or more globally in the corpus callosum by consumption of the oligotoxin cuprizone. Our findings show for the first time that PAR1 loss of function (Switch Off) enhances the replenishment of OPCs in each model, along with their differentiation toward a myelinating phenotype. Also, whereas in vitro studies suggest PAR1 loss of function in OPCs alone is sufficient to enhance differentiation, we provide new evidence that PAR1 also regulates the promyelinating properties of astrocytes. First, in the context of myelin injury PAR1 knock-out mice show increases in markers of astrocyte abundance, but reductions in astrocytic proinflammatory signatures. In conjunction with these findings, astrocyte cultures with PAR1 loss of function support improved OPC differentiation, in part by a TrkB-dependent mechanism. Altogether, these studies identify PAR1 as a new regulator of myelin regeneration whose activities are integrated across glial compartments and linked by growth factor regulatory systems. These data have implications for the design of new treatment strategies targeting the neurological sequelae of CNS demyelinating conditions.

\section{Materials and Methods}

Mice

Mice genetically deficient in PAR1 (PAR1 ${ }^{-/-}, \mathrm{B} 6.129 \mathrm{~S} 4-F 2 r^{\mathrm{tm} 1 \mathrm{Ajc}} / \mathrm{J}$, \#002862) have been back crossed to C57BL/6J mice (\#000664) for $>50$ generations (Burda et al., 2013; Radulovic et al., 2016; Choi et al., 2018). All mice were obtained from The Jackson Laboratory. Age-matched C57BL/6 $\left(\mathrm{PAR}^{+/+}\right)$mice served as controls. Experiments were performed using male mice to avoid gender-dependent heterogeneity of the lysolecithin (Taylor et al., 2010) and cuprizone models (Bielecki et al., 2016; Vega-Riquer et al., 2019) and to facilitate group housing. All animal experiments were carried with adherence to the National Institutes of Health's guidelines for animal care and safety and were approved by the Mayo Clinic Institutional Animal Care and Use Committee. All experimental procedures and downstream analyses were performed without knowledge of genotype.

\section{Lysolecithin model of focal demyelination}

Focal demyelination was induced by injecting lysophosphatidylcholine (L-4129, Sigma-Aldrich) into the white matter of the ventral spinal cord of 10 -week-old PAR $1^{+/+}$or PAR $1^{-1-}$ male mice (25-30 g). In each case, mice were anesthetized with ketamine $(100 \mathrm{mg} / \mathrm{kg}$, Fort Dodge Animal Health) and xylazine (12.5 mg/kg, Akorn) administered intraperitoneally. A dorsal laminectomy was performed at the thoracic 10th vertebra using microscopic guidance. The dura mater was pierced and a volume of $2 \mu \mathrm{l}$ of $1 \%$ solution of lysophosphatidyl choline (with $0.01 \%$ Evan's blue; L-4129, Sigma-Aldrich) in saline was injected into the spinal cord ventral funiculus using a $40 \mu \mathrm{m}$ glass micropipette at a rate of $0.2 \mu \mathrm{l} / \mathrm{min}$ using a stereotaxic microinjection system (Stoelting). The coordinates for microinjection were mediolateral $+0.3 \mathrm{~mm}$ relative to the dorsal median sulcus and dorsoventral $-1.4 \mathrm{~mm}$ relative to the dorsal surface. Postoperative care provided for $3 \mathrm{~d}$ to minimize discomfort included injection of sterile saline ( $0.2 \mathrm{ml} / \mathrm{d}$, i.p.), antibiotic (Baytril $50 \mathrm{mg} / \mathrm{kg} / \mathrm{d}$, i.p.), and analgesic (buprenorphine $0.05 \mathrm{mg} / \mathrm{kg} / \mathrm{d}$, s.c.)

Following a 14 or $28 \mathrm{~d}$ period of recovery, animals were deeply anesthetized with sodium pentobarbital $(250 \mathrm{mg} / \mathrm{kg}$, i.p.) and perfused with $4 \%$ paraformalydehyde (PFA, $\mathrm{pH} 7.2$ ). The $2 \mathrm{~mm}$ of spinal cord rostral to the injection site was embedded in paraffin and $6 \mu \mathrm{m}$ sections prepared for immunochemical analysis of the lesion cellular microenvironment. To quantify axon remyelination, the $2 \mathrm{~mm}$ of spinal cord caudal to the injection site was osmicated ( $1 \%$ osmium tetroxide, 0223B, Polysciences) and embedded in Araldite (18050, Ted Pella). One-micrometer semithin sections were cut from Araldite blocks and stained with 4\% p-Phenylenediamine (P6001, Sigma-Aldrich) to identify myelin sheaths. Images throughout the site of focal demyelination were taken under oil immersion at $60 \times$ (Olympus BX51 microscope) and montaged to visualize the complete lesion in Adobe Photoshop. The total lesion area was measured and counts of remyelinated axons (defined as lightly stained and thin myelin sheaths) were expressed as the number of remyelinated axons per square millimeter of lesion (Yoon et al., 2017).

The region of interest for quantification of cellular aspects of focal demyelination and remyelination in the lysolecithin model was determined using the overall neuropathological changes (with H\&E staining) to identify the lesion location, MBP loss to determine the lesion core, and astrogliosis (GFAP) and microglia activation (Iba1) to determine the lesion border. Lesion areas of $<20,000 \mu \mathrm{m}^{2}$ or $>70,000 \mu \mathrm{m}^{2}$ were excluded from the analysis. Sections showing obvious tissue damage due to injection were also excluded from the analysis. Tissue damage was defined as excessive axon spheroids, extensive glial scarring and inflammation that are not typically part of the lysolecithin model. These criteria resulted in 2-3 mice being excluded per group for a final number of PAR $1^{+/+} n=7$ at $14 \mathrm{~d}$ and $n=9$ at $28 \mathrm{~d}$; PAR $1^{-1-} n=8$ at $14 \mathrm{~d}$ and $n=$ 10 at $28 \mathrm{~d}$.

\section{Cuprizone model of demyelination and remyelination}

For cuprizone experiments, 10 - to 12 -week-old male mice (PAR1 ${ }^{+/+}$ and PAR $1^{-1-}$ ) were fed with a $0.3 \%$ cuprizone diet (Teklad Global Rodent Diet, TD.140805, Harlan Laboratories). Fresh cuprizone diet was provided every other day. Age-matched untreated controls were fed regular chow (5053, LabDiet). After 6 weeks, cuprizone feeding was discon- 
tinued and all mice continued on a regular diet. Mice were weighed every 3 weeks throughout the study. Mice were deeply anesthetized and either perfused for immunohistochemical (IHC) analysis after 6 weeks or returned to a regular diet and allowed to recover for 3 or 6 weeks before perfusion and tissue harvest. Each group consisted of between 4 and 8 male mice per time point.

Quantification of demyelination and remyelination in the cuprizone experiments focused on the corpus callosum since it is extensively demyelinated after cuprizone consumption. All animals fed cuprizone for 6 weeks showed extensive loss of MBP in the corpus callosum and all animals that entered the study were included in the final analysis. For cuprizone studies, two brain slices were taken at either $-0.22 \mathrm{~mm}$ or $-0.70 \mathrm{~mm}$ relative to bregma, embedded in paraffin and sectioned at 6 $\mu \mathrm{m}$ for downstream cellular analysis. All means and SEM shown represent the mean taken from both rostral and caudal sections of corpus callosum. Stained tissue sections were captured digitally at $20 \times$ on an Olympus BX51 microscope and using a DP72 or XM10 camera equipped with CellSens software 1.9 (Olympus) under constant illumination. Raw optical density (ROD) readings of immunoreactivity were determined for each antigen and expressed as a percentage of the total corpus callosum area quantified, which was a mean of $0.20 \pm 0.01 \mathrm{~mm}^{2}$ across animals. Image analysis was performed using ImageJ.

\section{Rotarod assay}

The accelerating rotarod test, measures overall balance, motor coordination and motor learning and has been found to be a sensitive predictor of cuprizone-mediated demyelination (Karamita et al., 2017). Mice were trained on the rotarod and a baseline collected. Mice were retested on the rotarod every 3 weeks for the duration of the experiment. During each testing session, mice experienced three trials of $300 \mathrm{~s}$ of increasing rod speed ( 7 to 50 r.p.m). A resting time of $60 \mathrm{~s}$ was allowed between each trial. The trial ended when the mouse fell off the rod or after reaching the cutoff time of $60 \mathrm{~s}$. The latency to the first fall was recorded.

\section{Histochemistry}

Immunochemical analyses of cellular changes across the lysolecithin and cuprizone models was performed on paraffin embedded materials as previously detailed (Radulovic et al., 2016; Yoon et al., 2017). Briefly, sections were deparaffinized followed by antigen retrieval (sodium citrate, $10 \mathrm{~mm}, \mathrm{pH} 6.0$ ), blocked with serum with or without $0.25 \%$ Triton $\mathrm{X}-100$ followed by incubation with primary antibodies overnight. For immunochemistry, sections were subsequently incubated with an appropriate biotinylated secondary antibody (Jackson Laboratories, 1:200) and developed using avidin biotin immunochemistry (Vectastain Elite ABC kit, PK-6100, Vector Laboratories) with 3,3' -diaminobenzidine tetrahydrochloride hydrate (DAB; D5637, Sigma-Aldrich) as the substrate. Nuclei were counterstained with methyl green (H-3402, Vector Laboratories) and coverslipped with DPX Mountant (06522, Sigma-Aldrich). For immunofluorescence staining, appropriate fluorochrome-conjugated secondary antibodies (donkey anti-mouse Alexa Fluor 488 and $555 \mathrm{~nm}$; donkey anti-rabbit Alexa Fluor 488, 555, and $647 \mathrm{~nm}$; and donkey antichicken Alexa Fluor 488 and $555 \mathrm{~nm}$ ) were applied after primary antibodies and 4', 6' -diamindino-2-phenylindole (DAPI, Vector Laboratories) was used as nuclear stain.

Antibodies. The following primary antibodies were used: C3 d (1:500, AF2655, R\&D Systems), CC-1 (1:50, OP80, Calbiochem), CD68 (1:1000, ab125212, Abcam), CD163 (1:100, ab111250, Abcam), GFAP (1:5000, Z0334, Dako), Ibal (1:3000, 019-19741, Wako), Ki67 (1:25, 550609, BD PharMingen), MBP (1:750, MAB386, Millipore), Neurofilament $\mathrm{H}$ $\left(\mathrm{NF}_{\mathrm{H}} 200,1: 1300\right.$, N4142, Sigma-Aldrich), rabbit-Olig2 (1:500, AB9610, Millipore), mouse-Olig2 (1:200, MABN50, Millipore), PAR1 (1:100, sc13503, Santa Cruz Biotechnology), PLP (1:750, ab28486, Abcam), and S100a10 (1:7500, 11250-1-AP, Proteintech).

\section{Cell culture}

Primary oligodendrocyte or astrocyte cultures were purified from mixed glial cultures prepared from the cortices of postnatal day 1 to 3 mice as described previously (Radulovic et al., 2016; Yoon et al., 2017). In all cases, mixed glia were grown in Dulbecco's modified Eagle's medium (DMEM) (11960-044, Invitrogen), containing $1 \mathrm{~mm}$ sodium pyruvate
(11360070, Corning), 20 mм HEPES (15630-080, Invitrogen), 10\% heatinactivated fetal bovine serum (Atlanta Biologicals), $100 \mathrm{U} / \mathrm{ml}$ penicillin and $100 \mu \mathrm{g} / \mathrm{ml}$ streptomycin (15140122, Life Technologies). Oligodendrocyte precursors and microglia were obtained from mixed cultures at $10 \mathrm{~d}$ in vitro by overnight shaking, leaving astrocytes at $\sim 95 \%$ purity (Radulovic et al., 2016). Oligodendrocyte progenitor cells were then purified from the retained supernatant by sequential panning on non-tissue culture-treated plastic to eliminate microglia and are $92-98 \%$ sulfatide positive after $24 \mathrm{~h}$ (Burda et al., 2013).

To determine the impact of PAR1 knock-out on oligodendrocyte progenitor cell (OPC) proliferation, cells were subcultured in expansion media as described by (Dugas and Emery, 2013) in the presence of 5-ethynyl-2'-deoxyuridine (EdU; $10 \mu \mathrm{M})$. OPCs were plated at $2 \times 10^{4}$ cells $/ \mathrm{cm}^{2}$ on poly-L-lysine (PLL, $10 \mu \mathrm{g} / \mathrm{ml} ;$ P1274, Sigma-Aldrich) coated $12 \mathrm{~mm}$ glass coverslips in DMEM-SATO base growth media containing $1 \times$ SATO $(10 \mathrm{mg} / \mathrm{ml}$ bovine serum albumin (A4503, SigmaAldrich), $10 \mathrm{mg} / \mathrm{ml}$ apo-transferrin (T1147, Sigma-Aldrich), $1.5 \mathrm{mg} / \mathrm{ml}$ putrescine (P5780, Sigma-Aldrich), $6 \mu \mathrm{g} / \mathrm{ml}$ progesterone (P8783, Sigma-Aldrich), and $4 \mu \mathrm{g} / \mathrm{ml}$ sodium selenite (S5261, Sigma-Aldrich)) in a final volume of DMEM (11960-044, Invitrogen) containing $100 \mathrm{U} / \mathrm{ml}$ penicillin and $100 \mu \mathrm{g} / \mathrm{ml}$ streptomycin (15140163, Invitrogen), $1 \mathrm{~mm}$ sodium pyruvate (25-000-CI, Corning), $5 \mu \mathrm{g} / \mathrm{ml}$ insulin (12585014, Invitrogen), $5 \mu \mathrm{g} / \mathrm{ml} \mathrm{N}$-Acetyl-L-cysteine (A8199, Sigma-Aldrich), $1 \times$ trace elements B (25-022-CI, Corning), $10 \mathrm{ng} / \mathrm{ml}$ D-biotin (B4639, Sigma-Aldrich), and 1× B27 (17504044, Sigma-Aldrich). Two hours after plating, media was additionally supplemented with $4.2 \mu \mathrm{g} / \mathrm{ml}$ forskolin (F6886, Sigma-Aldrich), $10 \mathrm{ng} / \mathrm{ml}$ ciliary neurotrophic factor (CNTF; NBP199451, Novus Biologicals), $10 \mathrm{ng} / \mathrm{ml}$ platelet-derived growth factor-AA (PDGF; H8291, Sigma-Aldrich), and $1 \mathrm{ng} / \mathrm{ml}$ neurotrophin-3 (NT-3; 450-03, PeproTech) along with EdU. All cells were maintained at $37^{\circ} \mathrm{C}$ and $5 \% \mathrm{CO}_{2}$. After a $72 \mathrm{~h}$ culture period cells were fixed with $3.7 \%$ formaldehyde in PBS, washed with $3 \%$ bovine serum albumin in PBS, and permeabilized with $0.5 \%$ Triton X-100. Cells were then incubated with the Click-iT reaction mixture (Alexa Fluor 555, Life Technologies) for detection of EdU labeling and colabeled for Olig2 immunoreactivity.

To determine the possible mechanism by which blocking PAR1 function improves myelin production in vitro, we first determined whether a PAR1 small-molecule inhibitor alters OPC differentiation. To accomplish this, purified PAR $1^{+/+}$OPCs were plated at $4 \times 10^{4} \mathrm{cells} / \mathrm{cm}^{2}$ on PLL coated glass coverslips in defined differentiation media as we previously described (Burda et al., 2013; Yoon et al., 2015). Defined OPC differentiation medium consisted of Neurobasal A media (10888022, Life Technologies) containing 1\% N2 (17502048, Life Technologies), 2\% B27 (17504044, Life Technologies), $100 \mathrm{U} / \mathrm{ml}$ penicillin and $100 \mu \mathrm{g} / \mathrm{ml}$ streptomycin (15140122, Life Technologies,), $1 \mathrm{~mm}$ sodium pyruvate (11360070, Corning), 0.45\% glucose (G8769, Sigma-Aldrich), 2 mm glutamax (35050-061, Fisher), 5\% BSA (A4503-100G, Sigma-Aldrich), 50 $\mu \mathrm{M} \beta$-mercaptoethanol (21-031-CV, Fisher), and $40 \mathrm{ng} / \mathrm{ml}$ T3 (T6397, Sigma-Aldrich). PAR $1^{+/+}$OPC cultures were treated with a PAR1 smallmolecule inhibitor, vorapaxar sulfate ( $100 \mathrm{~nm}$, Toronto Research Chemicals) applied alone or in combination with suboptimal levels of brain derived neurotrophic factor (BDNF, $1 \mathrm{ng} / \mathrm{ml}, 450-02$, PeproTech) for a $72 \mathrm{~h}$ period of differentiation.

To investigate the possibility that PAR1 loss of function in astrocytes may in turn affect OPC differentiation through indirect mechanisms, we used an astrocyte-OPC coculture system. PAR1 ${ }^{+/+}$astrocytes were plated on PLL coated glass coverslips at a density of $1.6 \times 10^{4} \mathrm{cells} / \mathrm{cm}^{2}$ in defined Neurobasal A differentiation media in the presence of vorapaxar $(100 \mathrm{~nm})$ for $18 \mathrm{~h}$, then switched to fresh Neurobasal A differentiation medium. PAR ${ }^{+/+}$OPCs $\left(4 \times 10^{4} \mathrm{cells} / \mathrm{cm}^{2}\right)$ were then added to wells containing astrocytes previously treated with vorapaxar or vehicle alone and cultured for $72 \mathrm{~h}$. In a second series of parallel experiments, we examined the impact of PAR $1^{+/+}$compared with PAR $1^{-1-}$ astrocytes on differentiation of added OPCs added to the astrocyte monolayer over the same $72 \mathrm{~h}$ period.

To determine the potential involvement of an astrocyte secreted factor in the promyelinating effects of PAR1 loss of function observed, supernatants from PAR $1^{+/+}$or PAR $1^{-/-}$astrocytes grown in Neurobasal A 
Table 1. Primers used for $q R T-P C R$

\begin{tabular}{ll}
\hline Gene & Primer sequence (forward/reverse) or company name and assay ID \\
\hline ALDH1L1 & Applied Biosystems; assay ID: Mm03048957_m1 \\
BDNF & Applied Biosystems; assay ID: Mm04230607_s1 \\
CD14 & IDT; assay ID: Mm.PT0.58.8871572.g \\
Clcf1 & IDT; assay ID: Mm.PT0.58.10279842 \\
GFAP & GCAGATGAAGCCACCCTGG/GAGGTCTGGCTTGGCCAC \\
H2D1 & IDT; assay ID: Mm.PT0.58.42136026.g \\
IGF1 & Applied Biosystems; assay ID: Mm00439560_m1 \\
IL-1 $\beta$ & IDT; assay ID: Mm.PT0.51.17212823 \\
IL-6 & Applied Biosystems; assay ID: Mm00446190_m1 \\
LIF1 & Applied Biosystems; assay ID: Mm00434762_g1 \\
S100a10 & IDT; assay ID: Mm.PT0.58.6571055 \\
STAT3 & Applied Biosystems; assay ID: Mm01219775_m1 \\
TGF- $\beta$ & Applied Biosystems; assay ID: Mm01178820_m1 \\
TNF $\alpha$ & Applied Biosystems; assay ID: Mm00443258_m1 \\
TSP1 & Applied Biosystems; assay ID: Mm00449032_g1 \\
Vim & IDT; assay ID: Mm.PT0.53a0.8720419 \\
\hline
\end{tabular}

differentiation media were collected at a $72 \mathrm{~h}$ time point and concentrated with Vivaspin 2 sample concentrators with a $100 \mathrm{kDa}$ molecular weight cutoff (GE28-9322-58, Sigma-Aldrich) followed by Vivaspin 2 sample concentrators with a $10 \mathrm{kDa}$ molecular weight cutoff (GE28-932247, Sigma-Aldrich) and the resulting $10 \times$ concentrated astrocyte conditioned media (ACM) was snap frozen until use. Protein concentrations were parallel across samples as determined by the bicinchoninic acid Protein Assay (Thermo Fisher Scientific). ACM was examined for its ability to promote OPC differentiation, when diluted to $1 \times$ in Neurobasal A differentiation media. The potential for BDNF to play a role in the ability of astrocytes-OPC cocultures, or ACM with PAR1 loss of function, to contribute to OPC differentiation was determined by the inclusion of the TrkB inhibitor ANA-12 (10 $\mu \mathrm{M}, 4781, \mathrm{R} \& \mathrm{D}$ Systems) in a subset of the wells.

All cell culture conditions were performed in triplicate and all experiments were repeated at least twice using independently derived cultures. All OPC differentiation experiments were followed for $72 \mathrm{~h}$ before fixation with $2 \%$ PFA and downstream immune localization of myelin associated markers, Olig2, PLP and MBP. Briefly, oligodendrocyte cultures were stained for MBP (1:750, MAB386, Millipore), PLP (1:750, Ab28486, Abcam) and Olig2 (1:200, MABN50, Millipore) using immunofluorescence, nuclei colabeled with DAPI (D1306, Thermo Fisher Scientific) and coverslipped with Fluoromount-G Mountant (0100-01, SouthernBiotech). Five random fields for each coverslip were captured digitally at $20 \times$ with an Olympus BX51 microscope and XM10 camera equipped with CellSens software 1.9 (Olympus) under constant illumination. ImageJ software was used to determine the PLP or MBP staining area per Olig ${ }^{+}$cell, as well as number of Olig2 or Olig ${ }^{+} / \mathrm{EdU}^{+}$cells.

$q R T-P C R$. qRT-PCR was used to determine changes in gene transcripts for astrocytes markers, cytokines and growth factors in primary astrocyte cultures from PAR $1^{+/+}$or PAR $1^{-/-}$. Primary astrocytes RNA was isolated using RNA STAT-60 (Tel-Test) and stored at $-80^{\circ} \mathrm{C}$ until the time of analysis. Amplification of the housekeeping gene Rn18s in all RNA samples was used to control for loading. qRTPCR amplification in each case was accomplished with an iCycler iQ5 system (Bio-Rad) using primers obtained from Integrated DNA Technologies or Applied Biosystems (Table 1).

\section{Statistical analyses}

All data are expressed as mean \pm SEM with analyses performed using SigmaPlot 13 (Systat Software). Comparisons between multiple groups were made using a one-way ANOVA or two-way ANOVA with NewmanKeuls (NK) post hoc test with degrees of freedom cited from the ANOVA tables generated in SigmaPlot. For pairwise comparisons between two groups a two-tailed unpaired Student's $t$ test was applied with degrees of freedom and $t$-statistics generated in SigmaPlot. All degrees of freedom, $t$ values and $\mathrm{F}$ statistics from these analyses are provided without Bonferroni correction (Armstrong, 2014). Statistical significance was set at $p<$ 0.05 . Quantification of results in all experiments was performed without knowledge of genotype.

\section{Results}

Blocking PAR1 improves spinal cord remyelination after lysolecithin-mediated focal demyelinating injury

To test whether PAR1 loss of function enhances myelin regeneration, we quantified the number of remyelinated axons and replenished oligodendrocytes at 14 and $28 \mathrm{~d}$ after focal injection of lysophosphatidylcholine (lysolecithin) into the ventral spinal cord white matter of adult male mice (Fig. 1). PAR1 knock-out mice showed 1.8-fold increases in the number of remyelinated axons at $14 \mathrm{~d}$ post injury (dpi) $(n=7, \mathrm{df}=15, t=-3.8, p=$ 0.002 , Student's $t$ test) and 1.4 -fold increases by $28 \mathrm{~d}(n=9, \mathrm{df}=$ $17, t=-2.4, p=0.03$, Student's $t$ test). Improvements in the number of remyelinated axons in PAR1 knock-out mice were accompanied by higher numbers of oligodendrocyte transcription factor $2^{+}\left(\right.$Olig2 $\left.^{+}\right)$cells at 14 (1.5-fold increases, $n=8, \mathrm{df}=$ $12, t=-4.24, p=0.001$, Student's $t$ test) and $28 \mathrm{dpi}$ (1.4-fold increases, $n=10$, df $=16, t=-2.17, p=0.04$, Student's $t$ test). In addition, the remyelinated lesions of PAR $1^{-1-}$ mice showed greater numbers of mature adenomatous polyposis coli protein ${ }^{+}$ $\left(\mathrm{CC}-1^{+}\right)$oligodendrocytes (1.4-fold, $n=9$, df $=18, t=-2.75$, $p=0.01$, Student's $t$ test) and improvements in the area covered by MBP-immunoreactivity at $28 \mathrm{dpi}$ ( 1.3 -fold, $n=9$, df $=14, t=$ $-2.11, p=0.05$, Student's $t$ test). No differences in the density of neurofilament staining were observed between genotypes. These results demonstrate that PAR1 loss of function promotes myelin regeneration after focal demyelinating injury of the adult CNS.

To address the potential impact of PAR1 knock-out on astrocyte and microglial function after lysolecithin demyelinating injury, we quantified both their reactivity and phenotypic state (Liddelow et al., 2017; Clarke et al., 2018) (Fig. 2). First, small but significant increases in the area immunoreactive for glial fibrillary acidic protein (GFAP) (Fig. $2 a$ ) were seen at 28 dpi in remyelinated lesions of PAR1 knock-out mice compared with wild-type controls ( $n=7, \mathrm{df}=14, t=-2.29, p=0.04$, Student's $t$ test). We then investigated any impact of PAR1 knock-out on glial proinflammatory or prorepair signatures (Liddelow et al., 2017) that are positioned to affect myelin regeneration. First, S100A10 (S100 calcium binding protein A10), a marker of a prorepair phenotype was 3.7-fold higher in $28 \mathrm{~d}$ remyelinating lesions in mice with PAR1 loss of function compared with wild-type mice $(n=7, \mathrm{df}=$ $14, t=-2.76, p=0.02$, Student's $t$ test). The abundance of C3d, a proinflammatory astrocyte marker, or CD68 and CD163, markers of proinflammatory and prorepair microglia, respectively, and the overall abundance of ionized calcium-binding adapter molecule 1 (Iba1) microglia, did not differ between genotypes in the lysolecithin model at any stage examined. These findings suggest that PAR1 loss of function promotes a prorepair microenvironment in the lysolecithin model by biasing astrocyte activation toward a prorepair state.

\section{Blocking PAR1 promotes signs of remyelination and preserves function in the cuprizone model}

Next, we tested whether genetic PAR1 loss of function also improves signs of myelin regeneration in a distinct in vivo model of remyelination elicited by cuprizone consumption (Fig. 3). Cuprizone is a copper chelator that causes toxicity and loss of oligodendrocytes in the corpus callosum resulting in a predictable pattern of demyelination after 6 weeks. Myelin repair then occurs spontaneously when mice are returned to regular chow (Matsushima and Morell, 2001). As expected based on our recent report (Choi et al., 2018), the density of Olig $2^{+}$cells in the corpus callosum of PAR $1^{-/-}$mice after 6 weeks on a cuprizone-free diet was 1.7 -fold greater than that of age-matched controls $(n=4, \mathrm{df}=8, t=5.38$, 
a

\begin{tabular}{|c|c|c|c|c|c|c|}
\hline & Group & $\begin{array}{c}\text { No. of } \\
\text { Animals }\end{array}$ & $\begin{array}{c}\text { No. of } \\
\text { Lesions }\end{array}$ & $\begin{array}{c}\text { Mean (Median) Lesion } \\
\text { Area }\left(\mathrm{mm}^{2}\right) \pm \text { SEM }\end{array}$ & $\begin{array}{c}\text { Mean (Median) } \\
\text { Remyelinated Axons/ } \\
\mathrm{mm}^{2} \pm \text { SEM }\end{array}$ & $\begin{array}{c}\text { Remyelination } \\
\text { vs PAR1+/+ } \\
\text { (P value) }\end{array}$ \\
\hline $14 \mathrm{dpi}$ & PAR1+/+ & $\begin{array}{l}7 \\
8\end{array}$ & $\begin{array}{l}7 \\
8\end{array}$ & $0.035 \pm 0.002(0.037)$ & $15,616 \pm 1,572(13,609)$ & 0.002 \\
\hline $28 \mathrm{dpi}$ & $\begin{array}{l}\text { PAR1+/+ } \\
\text { PAR1-/- }\end{array}$ & $\begin{array}{c}9 \\
10\end{array}$ & $\begin{array}{c}9 \\
10\end{array}$ & $\begin{array}{l}0.039 \pm 0.004(0.038) \\
0.044 \pm 0.005(0.041)\end{array}$ & $\begin{array}{l}36,492 \pm 3,579(28,670) \\
50,380 \pm 4,439(38,195)\end{array}$ & 0.03 \\
\hline
\end{tabular}

b

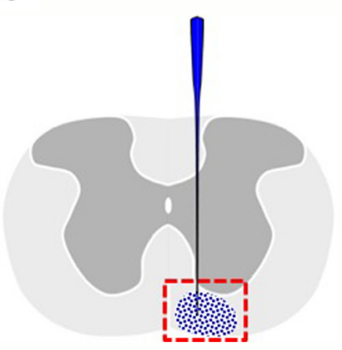

$1 \%$ LL $+0.01 \%$ Evans Blue

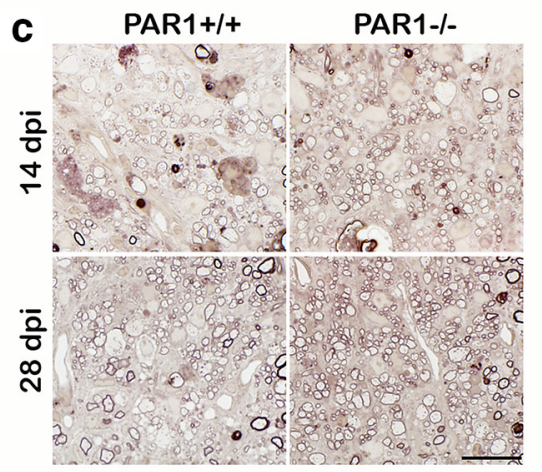

e PAR1+/+

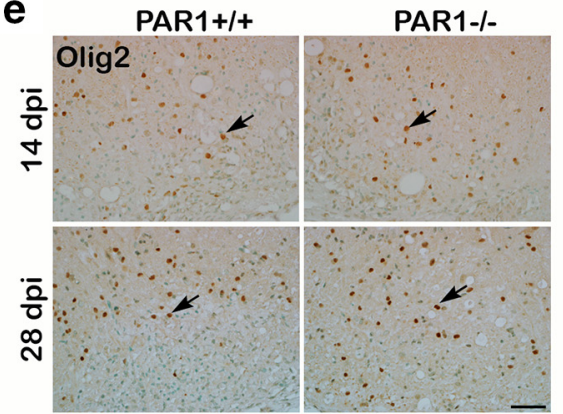

g

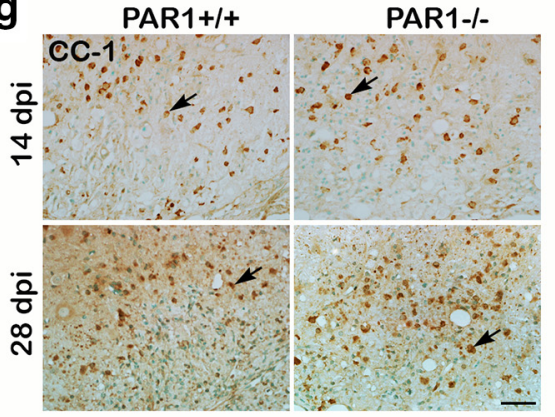

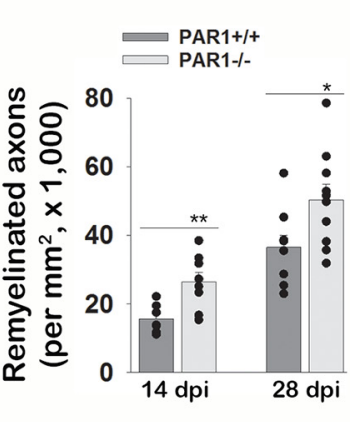

d

PAR1+/+

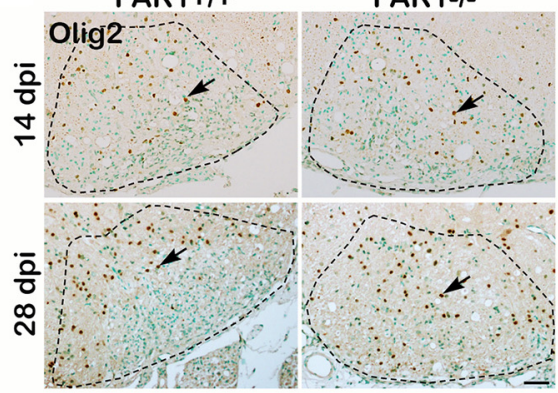

f

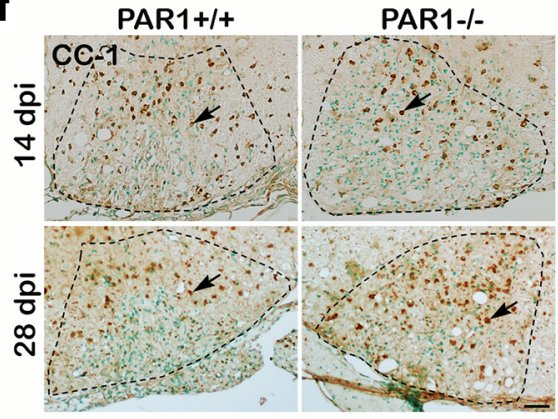

h

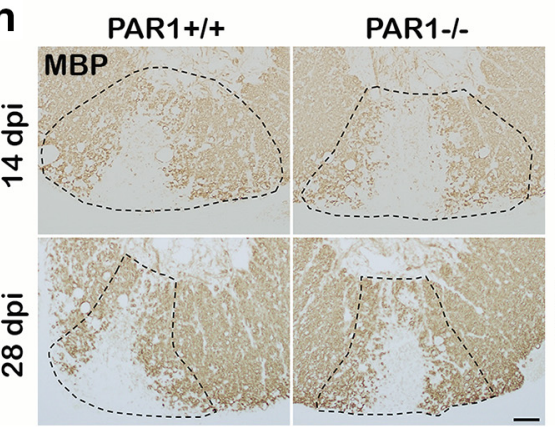

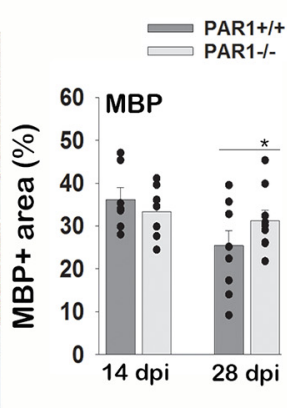

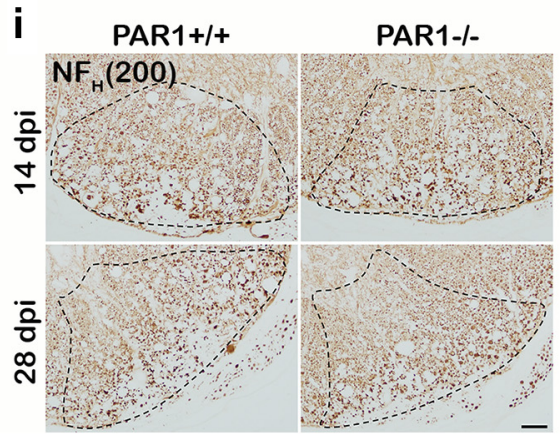

PAR $1+1+$

$\square$ PAR $1-$
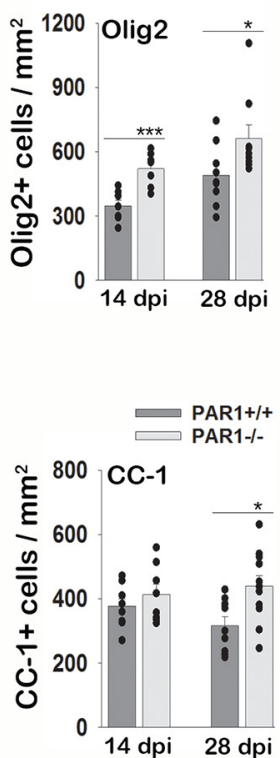

Figure 1. PAR1 knock-out improves myelin regeneration after focal lysolecithin demyelinating injury. $\boldsymbol{a}$, The mean number of remyelinated axons after focal lysolecithin-mediated demyelination of the ventral spinal cord white matter (diagram, $\boldsymbol{b}$ ) was greater in PAR1 knock-out mice at 14 or $28 \mathrm{~d}$ postinjury (dpi) ( $p=0.002$ and $p=0.03$, Student's $t$ test). $\boldsymbol{c}$, Images show representative paraphenylenediamine-stained thin sections from which counts of remyelinated axons were made. (d-f) PAR1 knock-out mice also showed improvements in the number of 0lig $2^{+}$oligodendrocyte lineage cells at 14 and $28 \mathrm{dpi}(p=0.001$ and $p=0.04)$ and increases in the number of $\left(C-1^{+}\right.$mature oligodendrocytes at $28 \mathrm{dpi}(p=0.01) . \boldsymbol{e}, \boldsymbol{g}$, Higher-magnification images of 0lig2 (d) and CC-1 (f) staining, with arrow indicating the same cell in the corresponding image. $\boldsymbol{h}$, By $28 \mathrm{dpi}$, higher levels of MBP were identified in the lesion area of PAR1 knock-out mice $(p=0.05) . \boldsymbol{I}$, , No significant changes were observed in neurofilament across time or genotype. Lesion boundaries quantified are indicated by a dotted line $(\boldsymbol{d}, \boldsymbol{f}, \boldsymbol{h}, \boldsymbol{i})$. Bar graphs represent mean \pm SEM of PAR $1^{+/+}$ $n=7$ at $14 \mathrm{~d}$ and $n=9$ at $28 \mathrm{~d} ;$ PAR $^{-1-} n=8$ at $14 \mathrm{~d}$ and $n=10$ at $28 \mathrm{~d}$. Asterisks in $d$ represent significant differences with ${ }^{*} p<0.05 ;{ }^{* *} p<0.01$; ${ }^{* * *} p \leq 0.001$, Student's $t$ test. Scale bars, $20 \mu \mathrm{m}(\boldsymbol{c})$ and $50 \mu \mathrm{m}(\boldsymbol{d}-\boldsymbol{i})$. 


\section{a}

\section{Astrogliosis}
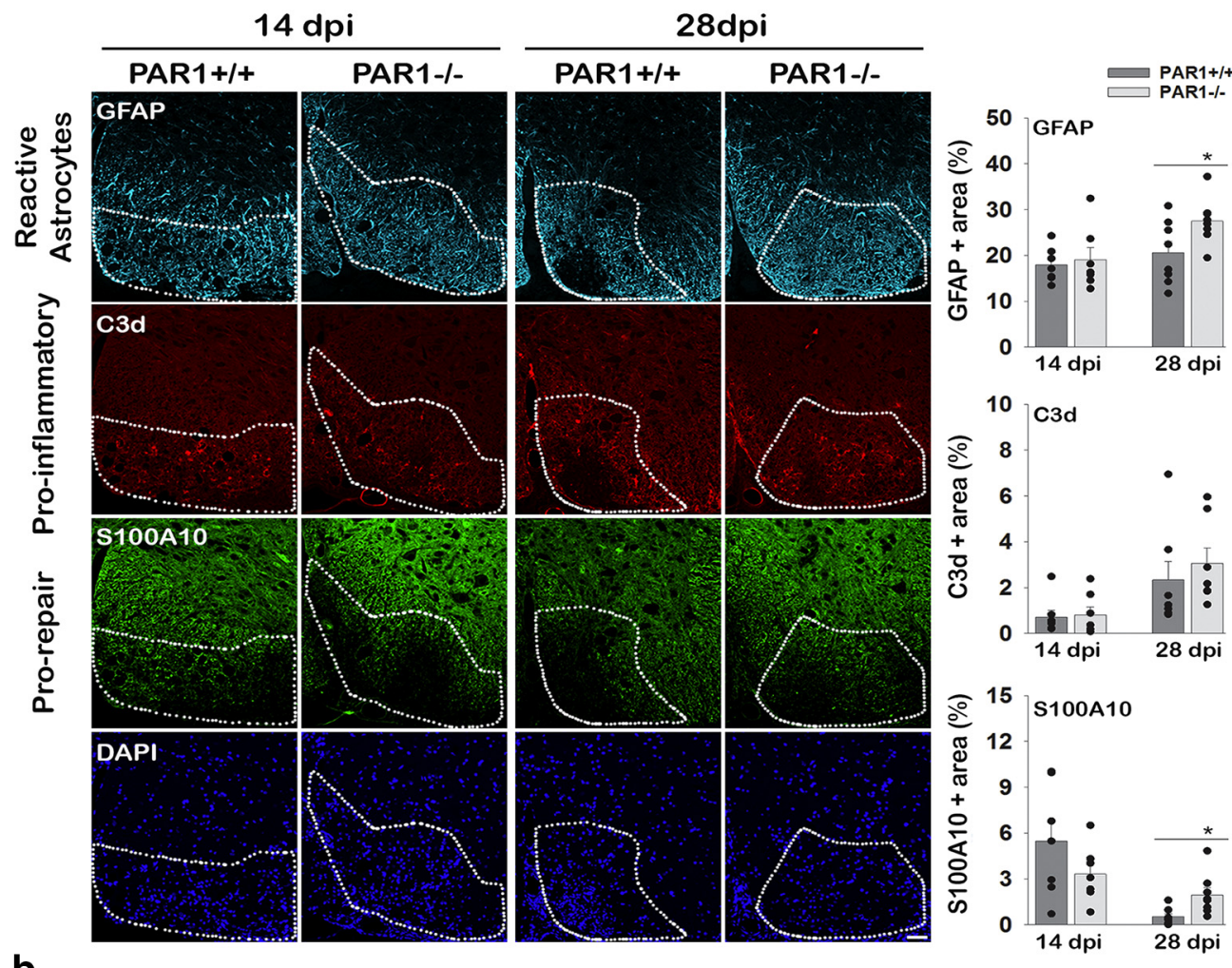

b

\section{Microgliosis}
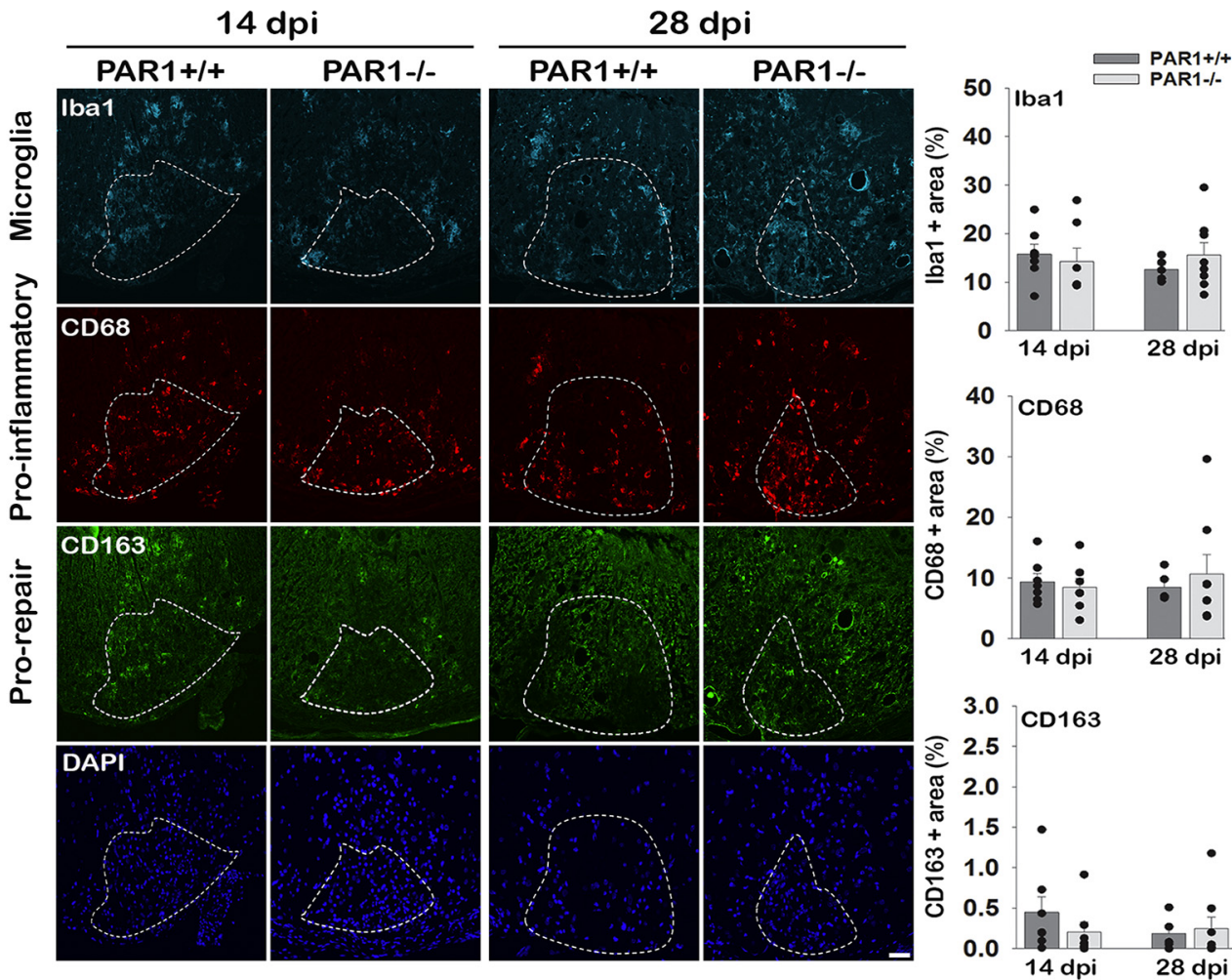

Figure 2. Enhanced remyelination after lysolecithin demyelination in PAR1 knock-out mice is accompanied by increases in astroglial prorepair signatures. $\boldsymbol{a}$, Immunofluorescence for GFAP (cyan) as a marker of astrogliosis showed higher levels in remyelinating lesions of PAR1 ${ }^{-1-}$ mice at $\left.28 \mathrm{dpi} \mathrm{(} p=0.04\right)$. Colabeling for an astrocyte marker considered prorepair (S100A10, green) was also elevated in remyelinating lesions of PAR1 knock-out mice at the same time point ( $p=0.02$ ), while the density of ( $3 \mathrm{~d}$ (red), a marker of proinflammatory astrocytes, did not differ across genotypes. $\boldsymbol{b}$, No genotype-related differences were observed in the density of immunofluorescence for $\mathrm{Iba} 1^{+}$microglia/monocytes (cyan) across the same time points, or in the associated proinflammatory (CD68) or prorepair markers (CD163, green). Shown are the means \pm SEM of PAR1 ${ }^{+/+}$and PAR1 ${ }^{-/-}$mice, $n=7$ at 14 and $28 \mathrm{~d}$ ). Asterisks represent significant differences with ${ }^{*} p<0.05$, Student's $t$ test. Scale bars in $\boldsymbol{a}$, and $\boldsymbol{b}$ indicate $50 \mu \mathrm{m}$. 
a

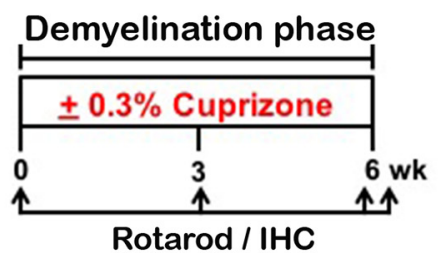

d
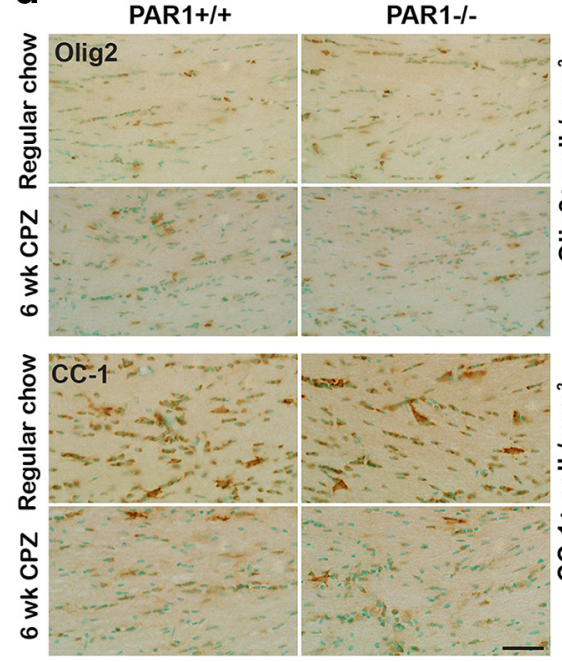

b

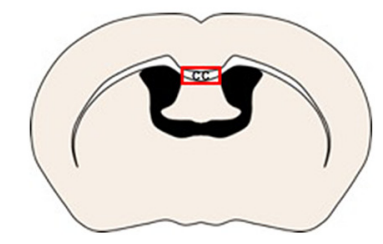

c

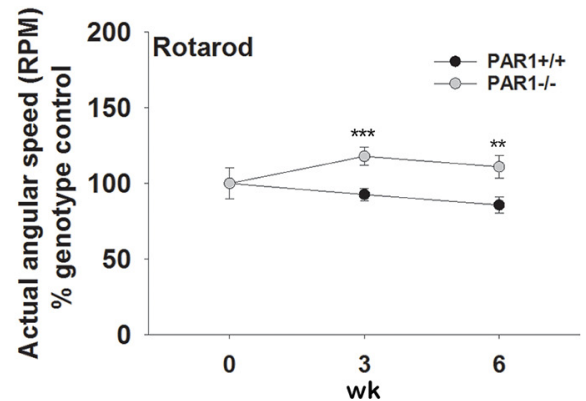

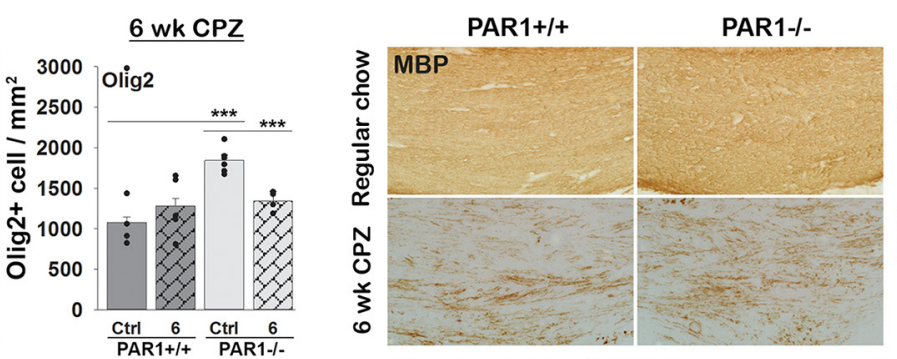

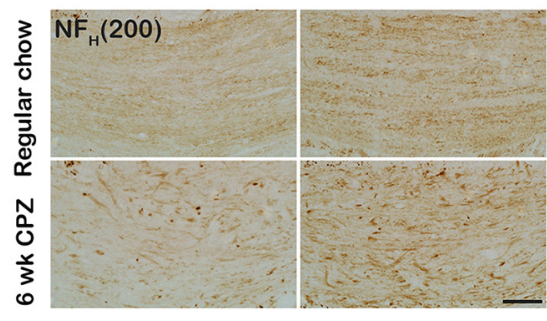

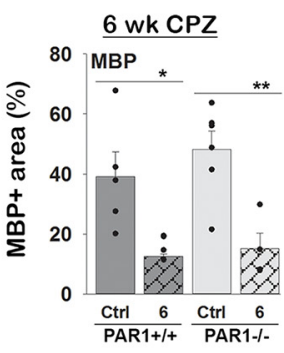

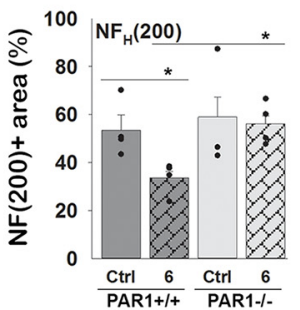

Figure 3. PAR1 knock-out does not alter cuprizone-mediated demyelination, but preserves neurofilament staining. $\boldsymbol{a}$, Schematic depicts the time course of demyelination during 6 weeks of cuprizone (CPZ) feeding, which predominantly occurs in the corpus callosum (CC) $\boldsymbol{b}, \boldsymbol{c}$, Motor function assessed by angular speed at fall on an accelerating rotarod test, expressed as a percentage of maximum at baseline for each genotype showed less (PZ-related deficit at 3 and 6 weeks of CPZ-feeding $(p=0.001$ and $p=0.006)$ in PAR1 ${ }^{-/-}$compared with PAR1 ${ }^{+/+}$. Arrows $\boldsymbol{a}$, show time points for rotarod assessment and immunohistochemistry (IHC) for markers of myelin injury and repair. $\boldsymbol{d}$, Representative images of the corpus callosum of wild-type or PAR1 knock-out mice fed regular chow (Ctrl) or (PZ laden chow for 6 weeks, with the corresponding quantification to the right. Although PAR1 ${ }^{-1-}$ mice showed higher numbers of oligodendrocyte lineage cells (0lig2 ${ }^{+}$) and mature oligodendrocytes $\left(\mathrm{CC}-1^{+}\right)$in the corpus callosum when fed regular chow $(p \leq 0.001)$, no differences in 0 lig $2^{+}$or $C\left(-1^{+}\right.$cell numbers, or in loss of MBP, were observed across genotypes after 6 weeks of CPZ consumption. The area positive for neurofilament in the corpus callosum at 6 weeks of (PZ feeding was greater in mice lacking PAR1 ( $p=0.007$ ). Statistical evaluation of rotarod $c$, was done by two-way ANOVA followed by NK post hoc test ( $p<0.003$ ( 3 weeks cuprizone) and $p<0.001$ ( 6 weeks cuprizone), $n=8$ across genotypes and diet conditions). Each bar in $\boldsymbol{d}$ represents the mean values for $n=4-5$ for each genotype across diet conditions with individual data points shown. Asterisks in $\boldsymbol{d}$ represent significant differences with ${ }^{*} p<0.05$; ${ }^{* *} p<0.01$; ${ }^{* * *} p \leq 0.001$, Student's $t$ test. Scale bar, $50 \mu \mathrm{m}$.

$p=0.0004$, Student's $t$ test). Despite the presence of more Olig2 ${ }^{+}$ cells in PAR $1^{-1-}$ mice at baseline, the absence of PAR1 did not alter MBP loss or the number of Olig ${ }^{+}$or $\mathrm{CC}-1^{+}$cells in the corpus callosum after 6 weeks of cuprizone-mediated demyelination. We observed 6 weeks of cuprizone consumption to cause a 3.1-fold reduction in immunoreactivity for MBP, and a 2.0-fold reduction in the number of mature $\mathrm{CC}-1^{+}$oligodendrocytes in both wild-type and PAR1 knock-out mice (Fig. $3 d$ ). Doublelabeling for Olig2 and Ki67 showed proliferation of Olig2 cells occurring during the period of cuprizone consumption and demyelination, but neither the counts of Olig2 ${ }^{+}, \mathrm{Ki} 67^{+}$or Ki67/ Olig2 double-labeled cells differed between wild-type and PAR1 knock-out mice after the 6 weeks period of cuprizone-mediated demyelinating injury (data not shown).

Six weeks of cuprizone consumption also resulted in a 1.6-fold loss of immunoreactivity for neurofilament 200 in wild-type mice ( $n=4, \mathrm{df}=6, t=2.97, p=0.03$, Student's $t$ test), an effect that was largely prevented in mice lacking PAR1 $(n=4, \mathrm{df}=6$, $t=4.06, p=0.007$, Student's $t$ test) (Fig. $3 d$ ). The reduction in immunoreactivity for neurofilament protein observed after 6 weeks of cuprizone-mediated demyelination persisted at similar levels across genotypes at 3 and 6 weeks of myelin repair (Fig. 4).
PAR1 knock-out mice showed improved signs of myelin regeneration at 3 and 6 weeks after returning mice to a cuprizonefree diet, thereby triggering innate repair mechanisms (Fig. 4). For example, PAR1 knock-out mice showed 1.2-fold higher numbers of Olig $2^{+}$oligodendrocytes after 3 weeks of remyelination ( $n=4-5, \mathrm{df}=7, t=-2.95, p=0.02$, Student's $t$ test). PAR1 knock-out mice also showed significant increases in the number of mature CC- $1^{+}$oligodendrocytes at the 3 weeks $(1.1$-fold, $n=$ $4-5, \mathrm{df}=6, t=2.05, p=0.05$, Student's $t$ test), and the 6 weeks (1.2-fold, $n=4$, df $=6, t=-2.44, p=0.02$, Student's $t$ test) intervals of myelin regeneration. Despite the improvements in myelinating cells in PAR $1^{-1-}$ compared with PAR $1^{+/+}$mice after 6 weeks of repair, levels of MBP (Fig. 4) and PLP (data not shown) immunoreactivity did not differ. In support of a potential impact of PAR1 knock-out on myelin loss during aging (Bouhrara et al., 2018; Nasrabady et al., 2018), there was also a perceptible loss in the relative abundance of $\mathrm{CC}-1^{+}$cells and MBP across the 12 weeks period of the experiment in wild-type mice fed regular chow, but these potential age-related reductions in myelin integrity were not observed or were significantly attenuated in mice lacking PAR1 $(n=4, \mathrm{df}=6, t=-3.67, p=0.01$ for $\mathrm{CC}-1^{+}$and $n=4, \mathrm{df}=6, t=-3.27, p=0.02$ for MBP, 
a

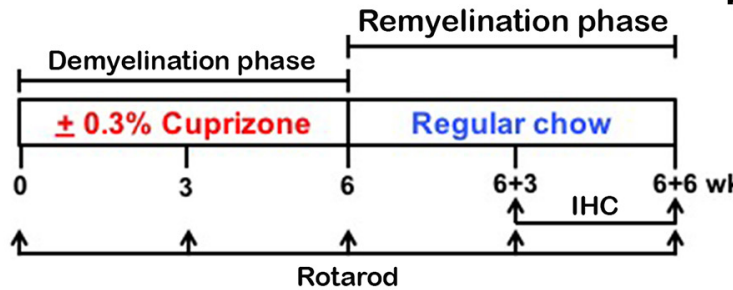

b

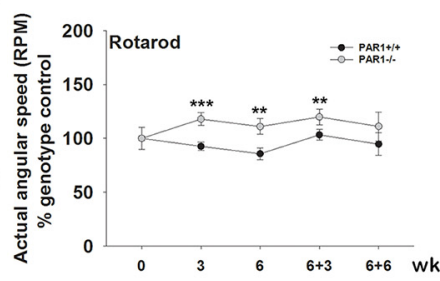

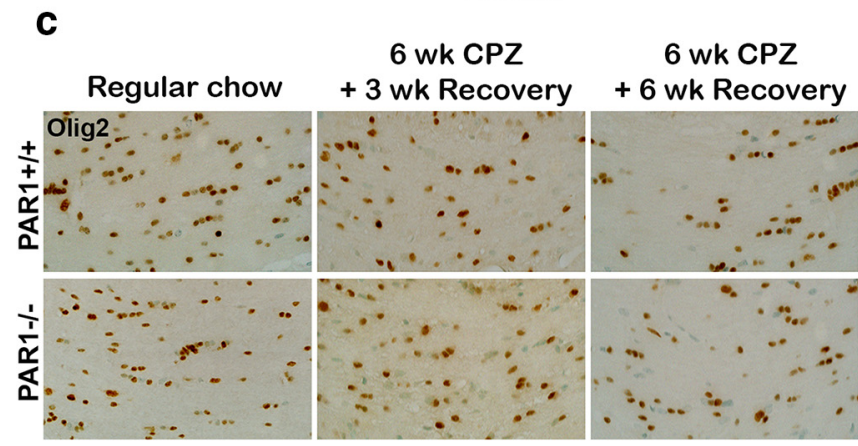

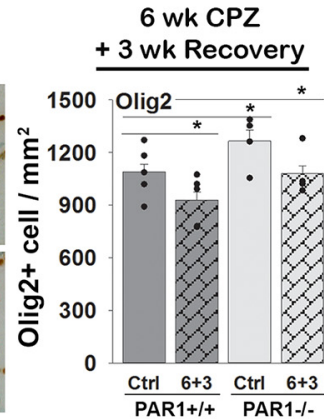

6 wk CPZ
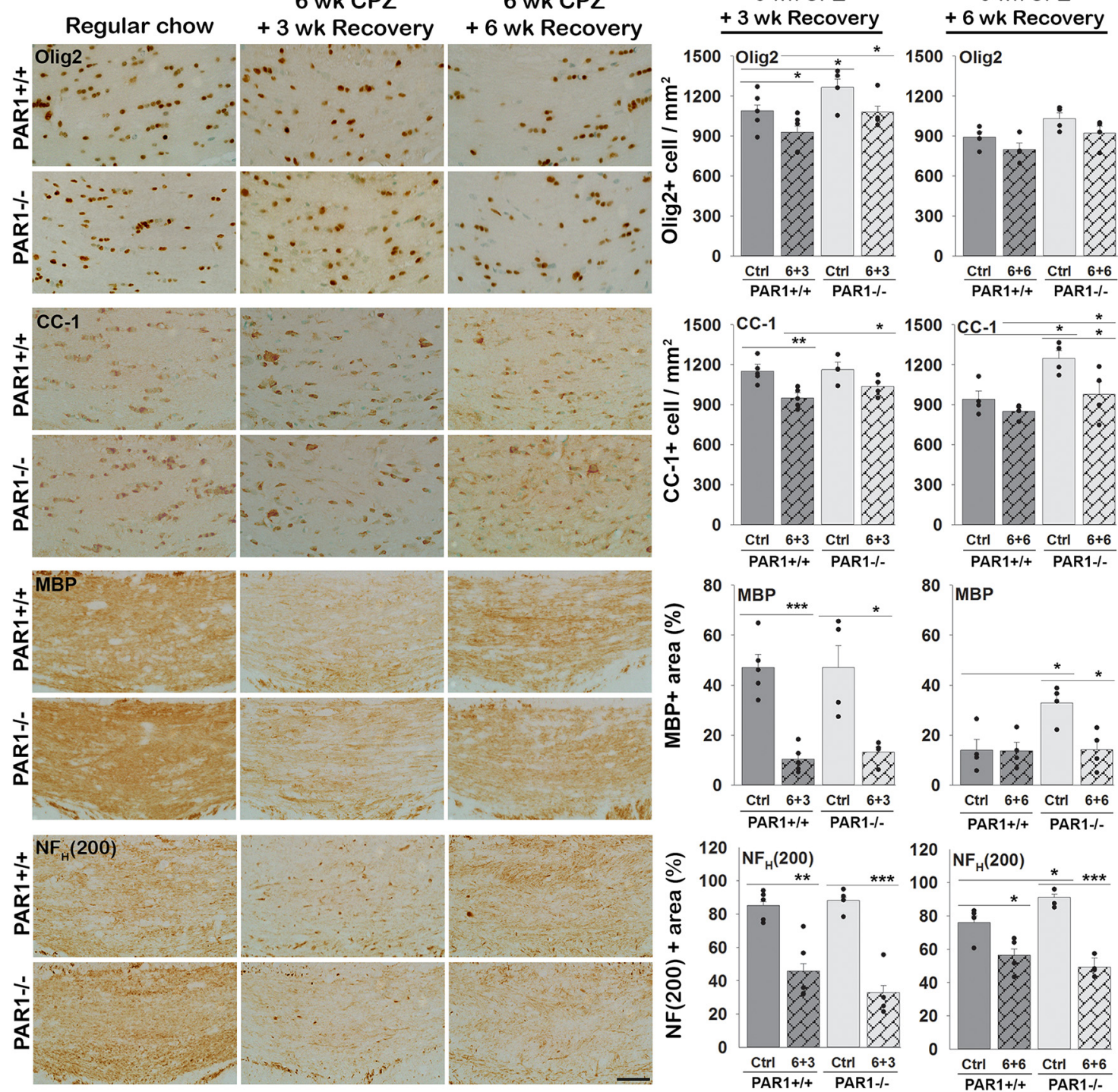

80 MBP
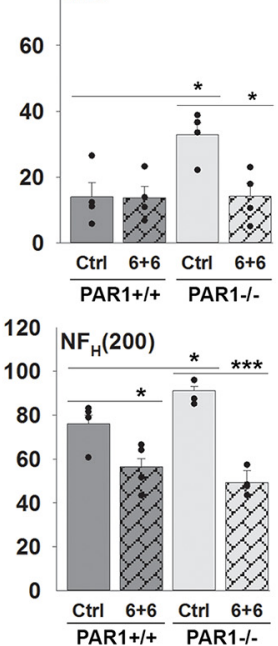

Figure 4. PAR1 knock-out improves signs of myelin regeneration after cuprizone withdrawal. $\boldsymbol{a}-\boldsymbol{c}$, Schematic depicting the phases of demyelination and remyelination during and after cuprizone (CPZ) feeding with arrows indicating time points for rotarod assessment $(\boldsymbol{b})$ and IHC for markers of myelin injury and repair (c). The impact of PAR1 knock-out on remyelination was assessed by feeding mice CPZ-laden chow for 6 weeks followed by a period of "induced remyelination" upon CPZ withdrawal and feeding regular chow for an additional $3(6+3)$ or $6(6+6)$ weeks period. $\boldsymbol{b}$, Motor function assessed by angular speed at fall on an accelerating rotarod test, expressed as a percentage of maximum at baseline for each genotype showed less $(\mathrm{PZ}$-related deficit at $3(p=0.001)$ and 6 weeks $(p=0.006)$ of CPZ-feeding and after an additional 3 weeks on regular chow $(6+3)$ in PAR1 knock-out mice $(p=0.003)$. c, Representative images through the corpus callosum of mice after 3 or 6 weeks of remyelination $(6+3$ or $6+6)$. Corresponding quantification of markers of remyelination showed PAR $1^{-1-}$ mice show greater increases in the number of oligodendrocyte lineage cells (Olig2 $\left.{ }^{+}\right)$after 3 weeks on regular chow and in the number of mature oligodendrocytes $\left(C\left(-1^{+}\right)\right.$at 3 and 6 weeks, compared with wild-type $(p \leq 0.05)$. The area stained for MBP or neurofilament (NF) after CPZ treatment was not altered by PAR1 knock-out. However, at the $6+6$ week time point PAR ${ }^{-1-}$ mice consuming regular chow for the full period of the experiment showed higher counts of $\mathrm{CC}-1^{+}$and higher levels of MBP and NF immunoreactivity in the corpus callosum $(p \leq 0.04)$ relative to regular chow WT counterparts. Statistical evaluation of motor function $(\boldsymbol{b})$ was done by two-way ANOVA followed by NK post hoc test (PAR1 ${ }^{-1-}$ effect $p<0.003$ ( 3 weeks cuprizone), $p<0.001$ ( 6 weeks cuprizone), $p=0.05$ ( 6 weeks cuprizone +3 weeks recovery), $n=4-8$ for each genotype). Each bar in c represents the mean value from $n=4-5$ for each genotype across diet conditions with individual data points shown, error bars $+/-$ SEM. Asterisks in c represent significant differences with ${ }^{*} p<0.05 ;{ }^{* *} p<0.01 ;{ }^{* * *} p \leq 0.001$, Student's $t$ test. Scale bar, $50 \mu \mathrm{m}$. 
a
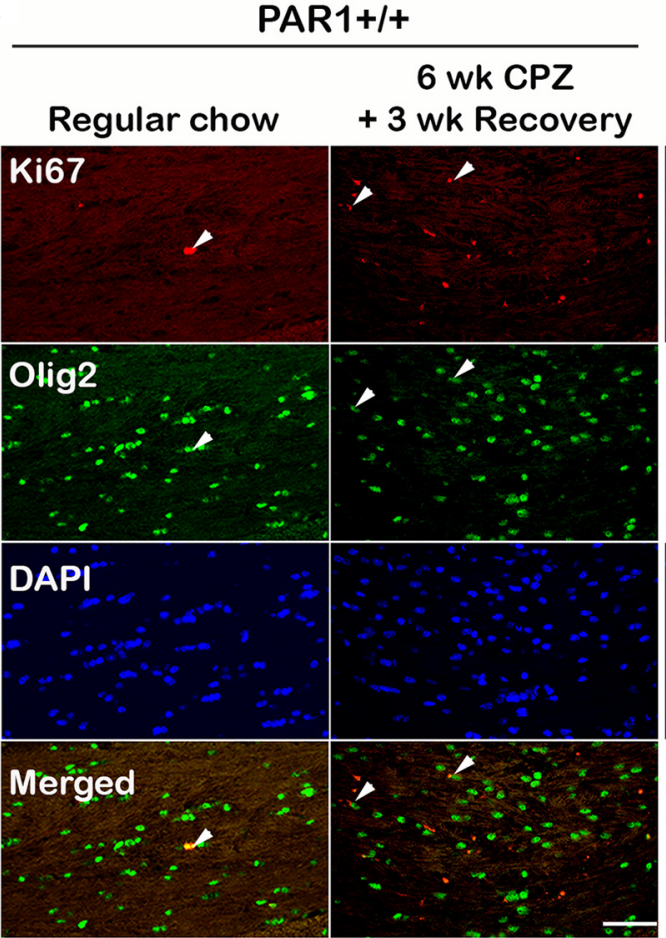

PAR1-/-

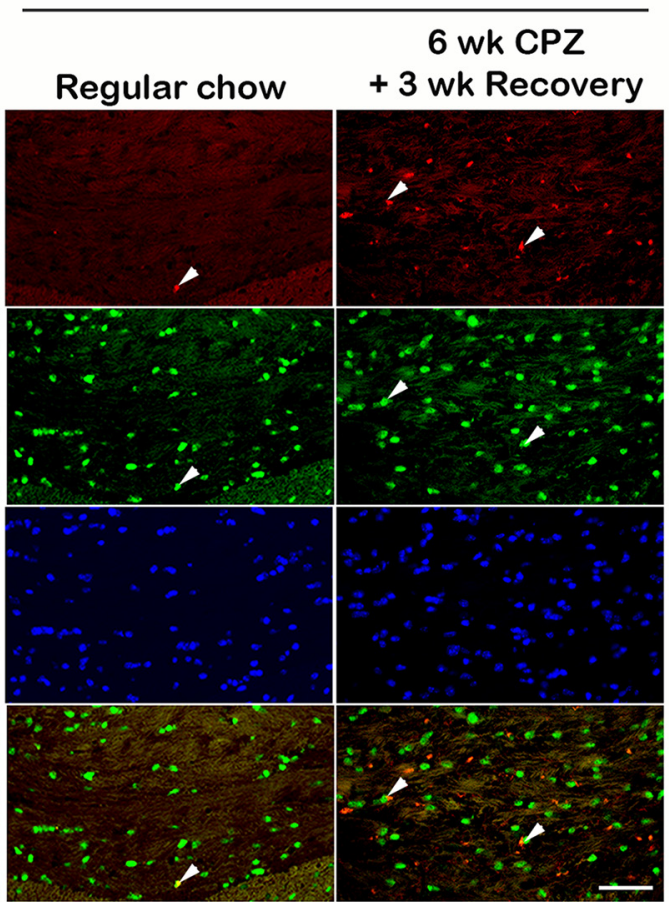

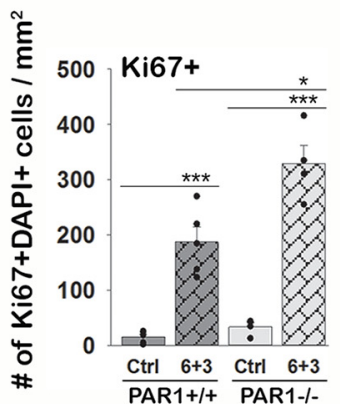

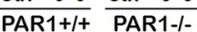

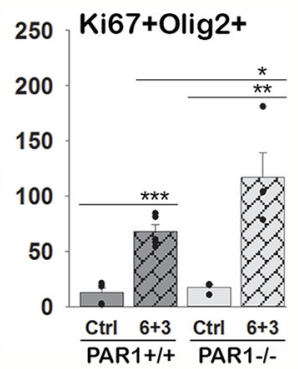

b
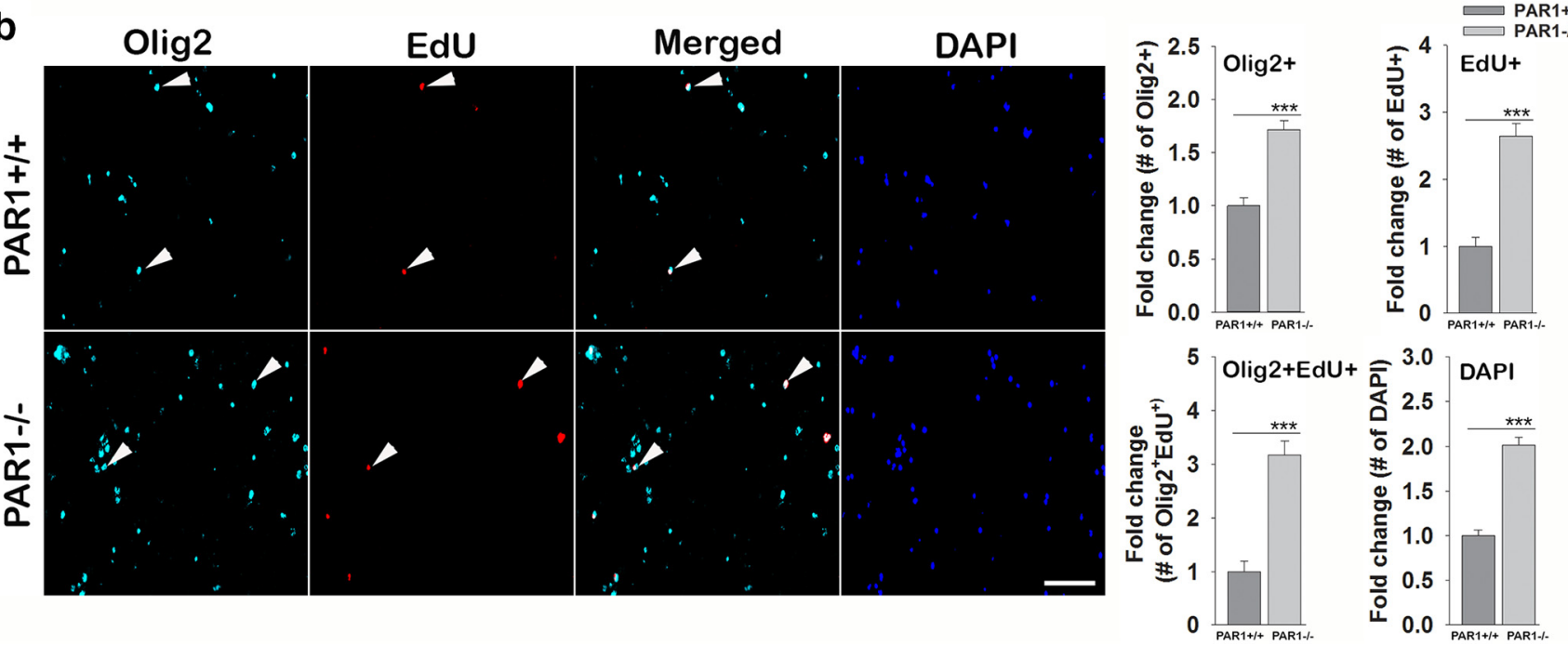

Figure 5. Enhanced remyelination after cuprizone-mediated demyelination in PAR1 knock-out mice is accompanied by increases in the number of proliferating oligodendrocyte lineage cells. $\boldsymbol{a}$, Representative images through the corpus callosum of mice after 6 weeks of $\mathrm{CPZ}$ feeding to induce demyelination followed by 3 weeks of feeding regular chow to elicit remyelination colabeled with Olig2 (green) for oligodendrocyte lineage cells and Ki67 (red) to identify proliferating cells. Corresponding quantification is on the right and demonstrates increases in proliferating oligodendrocyte lineage cells $\left(0\right.$ lig2 $\left.{ }^{+} \mathrm{Ki} 67^{+}\right) 3$ weeks after returning mice to regular chow, with this proliferative response occurring at increased levels in PAR1 ${ }^{-1-}$ mice compared with wild-type controls $(p=$ 0.05). Each bar in c represents the mean value from $n=4-5$ for each genotype across diet conditions with individual data points shown, error bars ${ }^{+/-}$SEM. $\boldsymbol{b}, 0$ ligodendrocyte progenitors purified from the cortex of PAR1 ${ }^{-1-}$ mice and grown for $72 \mathrm{~h}$ in culture, show enhanced incorporation of EdU (red) as a marker of proliferation, compared with parallel cultures derived from wild-type mice $(p=0.001)$. Arrows indicate a subset of 0 lig2 (cyan)/EdU double labeled cells in each case. Asterisks represent significant differences with ${ }^{*} p<0.05$; ** $p<0.01$; ${ }^{* * *} p \leq 0.001$, Student's $t$ test. Scale bars in $\boldsymbol{a}$, and $\boldsymbol{b}$ indicate $50 \mu \mathrm{m}$.

Student's $t$ test) (Fig. 4). PAR1 knock-out mice consuming regular chow also showed improved preservation of neurofilament staining in the corpus callosum over time. By the 12-week study end point, when mice were $\sim 24$ weeks old, PAR $1^{-1-}$ mice consuming regular chow showed 1.2-fold higher levels of neurofilament in the corpus callosum compared with PAR ${ }^{+/+}$mice ( $\mathrm{df}=6, t=-2.55, p=0.04$, Student's $t$ test, Fig. $4 c$ ).

To determine whether the improvements observed in Olig2 ${ }^{+}$ cell numbers at 3 and 6 weeks of remyelination and in $\mathrm{CC}-1^{+}$ cells at 6 weeks may reflect enhanced proliferation, sections were colabeled for Olig2 and Ki67, a marker of proliferation (Fig. 5a). At 3 weeks of induced repair, there was marked Olig2 proliferation in both genotypes (5.3-fold increases in wild-type and 6.6fold increases in PAR1 knock-out). Across genotypes, 1.7-fold higher levels of Olig2 proliferation occurred in PAR1 knock-out compared with wild-type mice $(n=4-5, \mathrm{df}=7, t=-2.36, p=$ 0.05 , Student's $t$ test). PAR $1^{-1-}$ OPC monocultures also showed an increase in the number of Olig $2^{+}$cells compared with parallel 
wild-type cultures with a coordinate increase in EdU incorporation pointing to an enhancement in proliferation (Fig. 5b). Together these findings suggest that enhancements in OPC proliferation in the context of PAR1 loss of function are positioned to contribute to the enhancements in myelin regeneration observed.

To investigate whether improvements in remyelination observed in PAR1 knock-out mice in the cuprizone model are associated with improved clinical measures, we assessed motor coordination and strength using an accelerated rotarod paradigm at baseline and at 3 weeks intervals during the periods of myelin injury and regeneration (Figs. $3 c, 4 b$ ). The angular speed that mice were able to maintain their balance on the rotarod improved to a greater extent in PAR1 knock-out mice during the period of demyelination and at 3 weeks after induced myelin repair (two-way ANOVA, genotype $F_{(1,99)}=13.7, p<0.001$; time point $F_{(4,99)}=1.8, p=0.133$; interaction $F_{(4,99)}=1.2, p=$ 0.31 ; and $p<0.003$ ( 3 weeks cuprizone), $p<0.001$ ( 6 weeks cuprizone), $p=0.05$ ( 6 weeks cuprizone +3 weeks recovery, $\mathrm{NK}), n=4-8$ per genotype depending on time after cuprizone examined). These findings suggest that PAR1 loss of function during the period of demyelination has the potential to preserve neurological function.

\section{PAR loss of function in the cuprizone model is associated with reductions in astrocyte and microglial proinflammatory properties}

Given the increases in $\mathrm{GFAP}^{+}$astrocytes in the CNS of PAR1 knock-out mice at baseline (Radulovic et al., 2016) (Fig. 6a), we sought to quantify differences in GFAP abundance during the phases of cuprizone-mediated myelin injury and induced repair. As expected, GFAP immunoreactivity was increased 7.4-fold in wild-type mice after 6 weeks of cuprizone-mediated demyelination, and parallel increases were observed in PAR1 knock-out mice. The significant increases in GFAP staining observed at 6 weeks after cuprizone-mediated demyelination across genotypes largely persisted at 3 and 6 weeks after cuprizone, though were slightly reduced in PAR1 knock-out mice. Increases in GFAP at 3 weeks of remyelination reached 4.2-fold in wild-type mice, but only threefold in mice lacking PAR1 ( $\mathrm{df}=7, t=2.96, p=0.02$, Student's $t$ test, $n=4-5$ ).

Since astrocytes play key roles in myelin regeneration (Nash et al., 2011; Hibbits et al., 2012; Skripuletz et al., 2013; Hammond et al., 2014), we also investigated astrocyte proinflammatory (Fig. $6 a$ ) or prorepair profiles (Fig. $7 a$ ) in the models examined. The proinflammatory marker Complement 3 (C3d) was not expressed at significant levels in the corpus callosum of wild-type or PAR $1^{-/-}$mice consuming regular chow (Fig. $6 a$ ). The area of C3d immunoreactivity was increased by 15 -fold in the corpus callosum of wild-type mice by the end of the 6 weeks period of cuprizone-mediated demyelination $(n=4-5$, $\mathrm{df}=5, t=-2.7$, $p=0.04$, Student's $t$ test), by 8 -fold after 3 weeks of myelin regeneration, and returned to very low baseline levels 3 weeks later. By contrast, increases in the proinflammatory astrocyte signature C3d were completely absent in PAR1 knock-out mice at all time points of cuprizone-mediated myelin injury and regeneration examined. The prorepair astrocyte marker S100A10 was 2.5fold higher at baseline in the corpus callosum of PAR1 knock-out mice with similar increases occurring at all stages of cuprizone injury and induced repair (Fig. 7a). These findings suggest that the beneficial effects conferred on myelin regeneration by blocking PAR1 may involve changes in astroglial responses that include a dampening of proinflammatory astrocyte reactivity.
Myelin regeneration in the cuprizone model is heavily influenced by microglia/monocyte activity (Miron et al., 2013; Lampron et al., 2015). Quantification of these responses in the current study showed 2.8-fold increases in the abundance of Iba1 after 6 weeks of cuprizone-mediated demyelination in the corpus callosum of wild-type mice (Fig. 6b). Notably, this increase in Iba1 was completely absent in PAR1 knock-out mice. We next considered whether the attenuated microglial responses observed in PAR $1^{-/-}$ mice after demyelination are reflected in differences in proinflammatory or prorepair signatures. First, a marker of proinflammatory microglia, CD68, was lower in PAR1 knock-out mice after 6 weeks of cuprizone-mediated demyelination and at 3 weeks of repair, although these differences were not statistically significant (Fig. $6 b, \mathrm{df}=7, t=1.46, p=0.19$, Student's $t$ test). CD163, a marker of prorepair microglia was elevated by 60 -fold in PAR $1^{+/+}$mice and by 190 -fold in PAR $1^{-/-}$mice at 3 weeks of remyelination (Fig. $7 b, \mathrm{df}=7, t=-2.74, p=0.03$, Student's $t$ test. These results demonstrate that the beneficial effects conferred by blocking PAR1 function on myelin regeneration include not only a reduction in microglial/monocyte abundance, but also a skewing of their properties toward a prorepair phenotype.

\section{Evidence that blocking PAR1 enhances myelin production through oligodendrocyte progenitor direct and OPC- astrocyte indirect-trophism}

To gain insights into the cellular mechanisms underlying the enhanced levels of myelin and improvements in myelin regeneration observed in PAR1 knock-out mice, we investigated the impact of PAR1 loss of function in purified cultures of OPCs or in coculture with astrocytes. First, to differentiate the potential contributions of OPC PAR1 to myelin production through possible direct actions we examined the influence of a PAR1 smallmolecule inhibitor, vorapaxar, applied alone or in combination with suboptimal concentrations of brain derived neurotrophic factor (BDNF) (1 ng/ml) (Fig. 8a). Supporting our prior report that OPC monocultures derived from PAR1 knock-out mice show enhanced signs of differentiation (Yoon et al., 2015), wildtype OPCs treated with vorapaxar for $3 \mathrm{~d}$ in vitro, showed a 1.2fold increase in the number of Olig ${ }^{+}$cells one-way ANOVA, $\mathrm{df}=3, F=10.5, p=0.007, \mathrm{NK})$ and a 1.6-fold increase in PLP expression per cell (one-way ANOVA, df $=3, F=12.4, p<$ $0.001, \mathrm{NK})$. In addition, the current studies demonstrate that increases in Olig2 and PLP observed with pharmacological inhibition of PAR1 mirrored those occurring following the application of low concentrations of BDNF. Of considerable therapeutic interest, we observed an additive effect when vorapaxar and low dose BDNF were applied jointly, in which case Olig2 numbers were increased by 1.5 -fold (one-way ANOVA, $\mathrm{df}=3, F=10.5$, $p=0.03, \mathrm{NK})$ and PLP expression per cell was increased by 1.9-fold (one-way ANOVA, df $=3, F=12.4, p=0.005, \mathrm{NK}$ ). These results demonstrate for the first time that a Food and Drug Administration (FDA)-approved small-molecule inhibitor of PAR1 enhances the myelinating potential of OPCs and also potentiates the effects of suboptimal BDNF.

To determine whether PAR1 loss of function in astrocytes affects their ability to support the myelinating potential of OPCs, we used pharmacologic (vorapaxar, Fig. 8b) or genetic (Fig. 9) approaches to block astrocyte PAR1 function and then determined their ability to regulate OPC numbers and their expression of PLP in an astrocyte-OPC coculture system (Fig. 8b). Both OPCs cocultured with astrocytes that had been pretreated with the PAR1 inhibitor vorapaxar, and OPCs cultured with condi- 


\section{a}

\section{Astrogliosis}
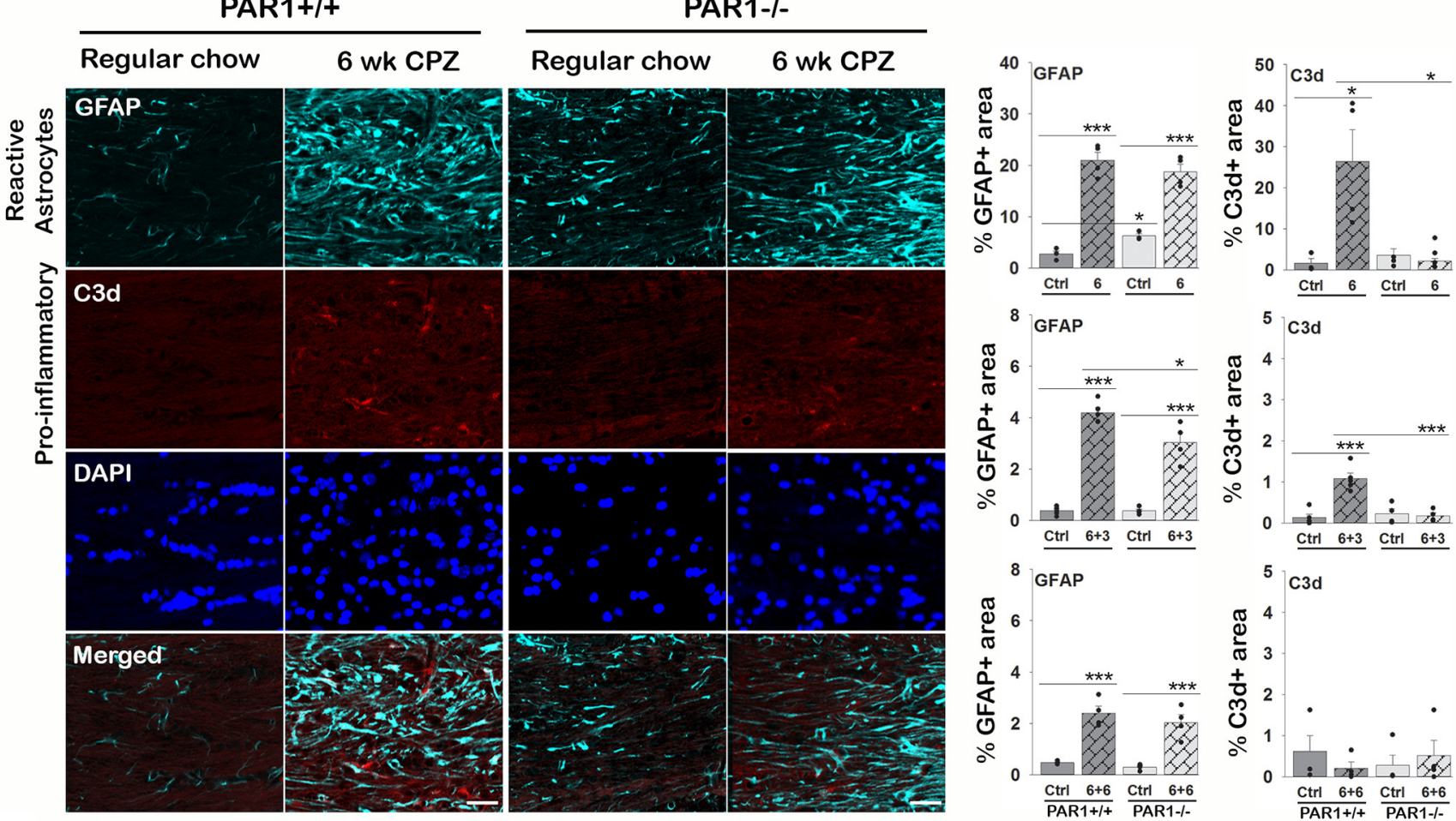

b

\section{Microgliosis}
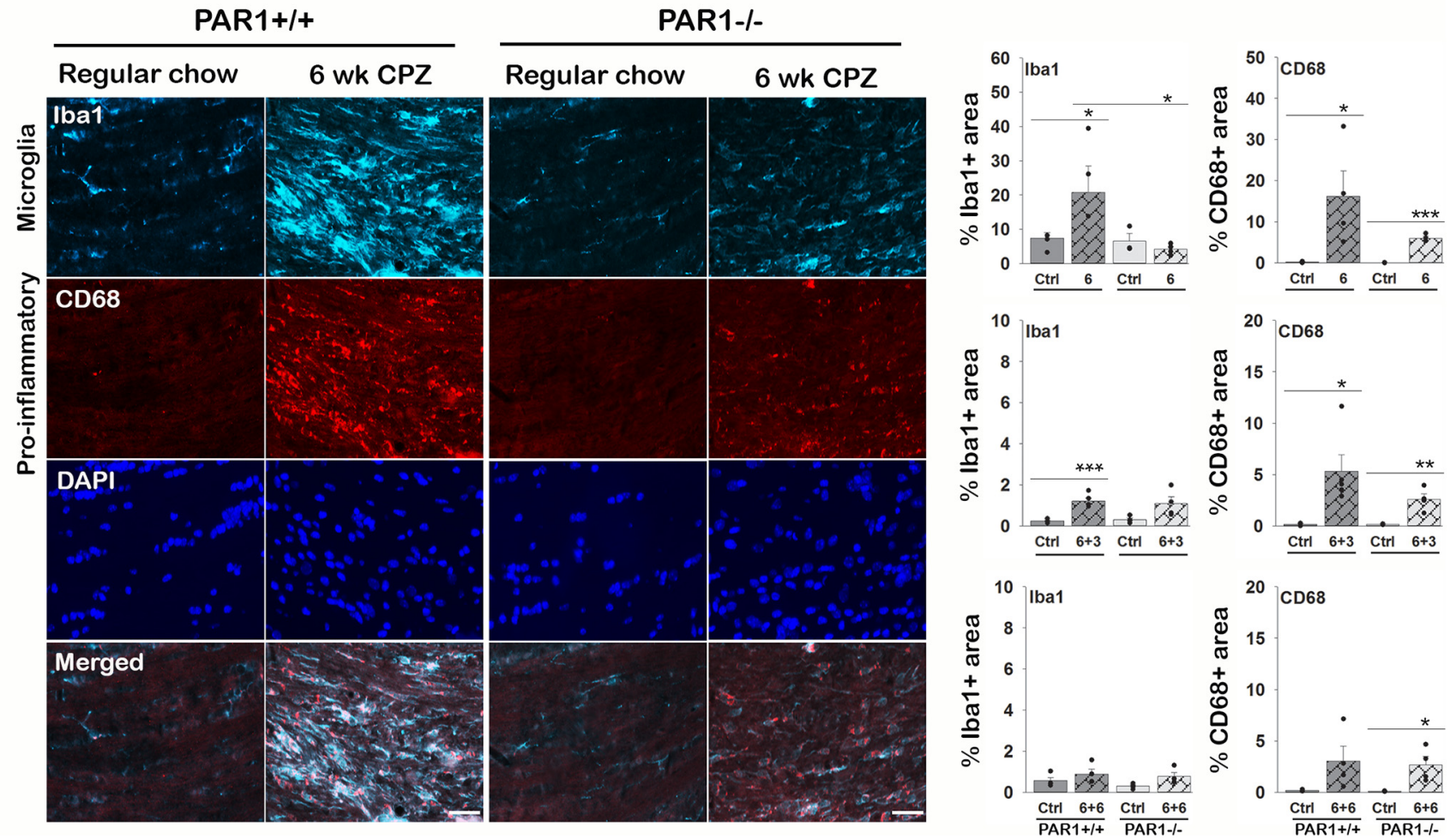

Figure 6. Enhanced remyelination after cuprizone demyelination in PAR1 knock-out mice is accompanied by reductions in astroglial and microglial proinflammatory signatures. $\boldsymbol{a}$, Representative images through the corpus callosum of mice after 6 weeks of cuprizone (CPZ) feeding to induce demyelination colabeled by immunofluorescence for reactive astrocytes using GFAP (cyan) and the astrocyte proinflammatory marker $(3 \mathrm{~d}(\mathrm{red})$. Corresponding quantification is on the right, and includes values for 3 weeks $(6+3)$ or 6 weeks $(6+6)$ of remyelination that occurs upon return of mice to regular chow. Significant increases in C3d at 6 weeks and at $6+3$ weeks were completely absent in mice lacking PAR1 $(p=0.03$ and $p=0.0009$ ). Mice lacking PAR1 also showed attenuated elevations in GFAP at $6+3(p=0.02)$, despite overall higher GFAP density in PAR1 ${ }^{-1-}$ mice consuming regular chow 6 weeks $(p=0.006)$. $\boldsymbol{b}$, Parallel sections were colabeled for a pan microglia/monocyte marker (Iba1, cyan) and a microglial proinflammatory marker CD68 (red). Prominent increases in Iba1 and CD68 were seen across genotypes after 6 weeks of CPZ

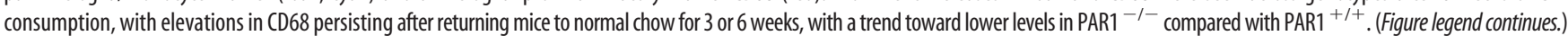




\section{Astrogliosis PAR1+/+}

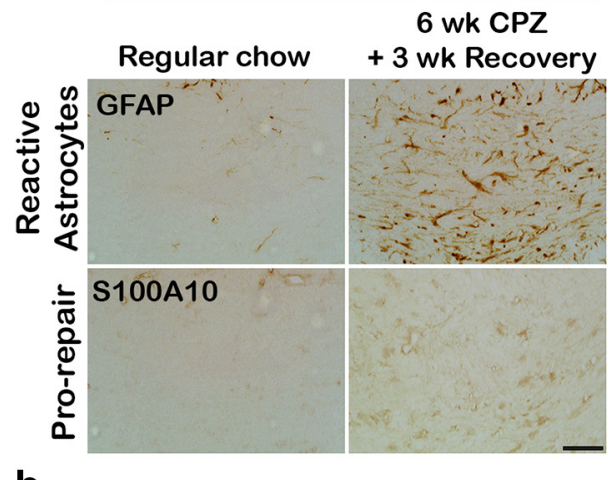

b
PAR1+/+

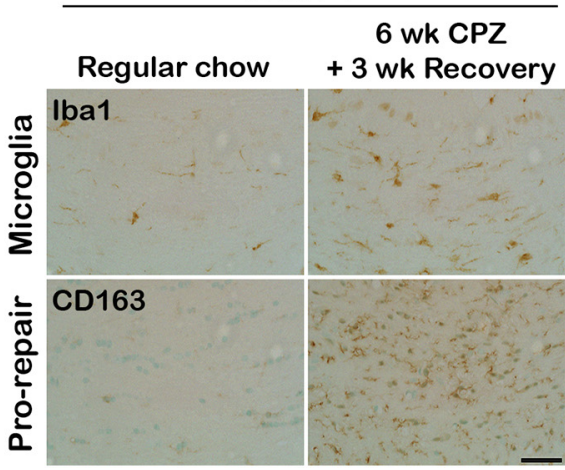

PAR1-1-

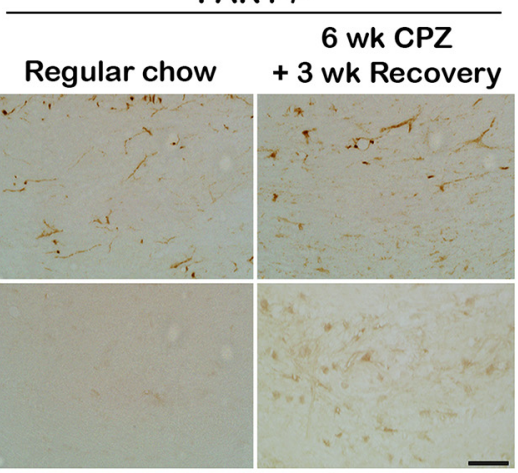

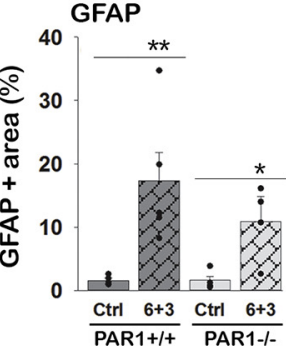

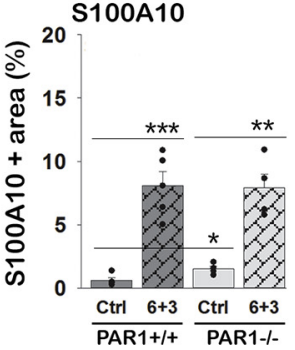

PAR1-I-
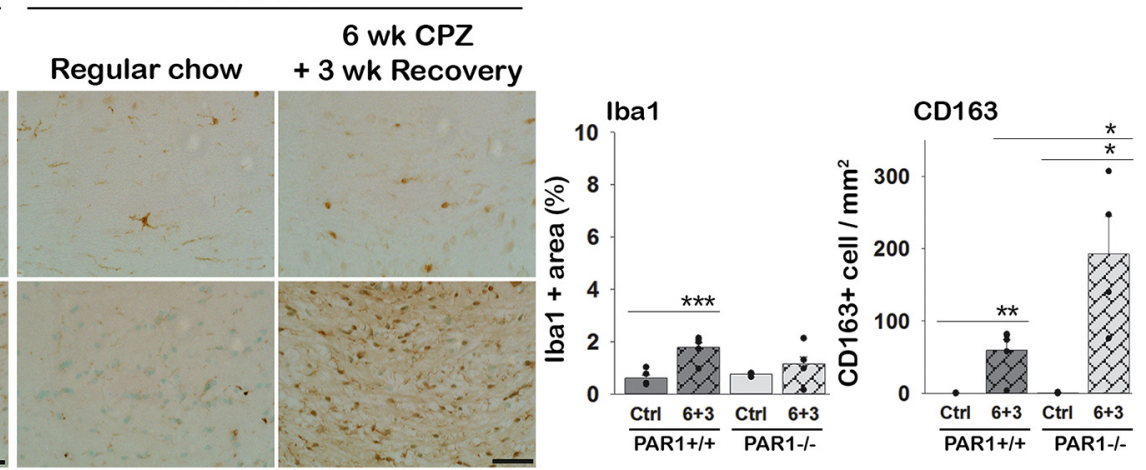

Figure 7. Enhanced remyelination after cuprizone demyelination in PAR1 knock-out mice is accompanied by increased prorepair microglial signatures. Images show immunostaining for reactive astrocytes (GFAP; $\boldsymbol{a}$ ) and microglia (Iba1; $\boldsymbol{b}$ ) in addition to markers of prorepair astrocytes (S100A10) or prorepair microglia/monocytes (CD163) in the corpus callosum of mice consuming regular chow or those that consumed cuprizone for 6 weeks followed by 3 additional weeks of regular chow to elicit myelin regeneration. Staining for prorepair astrocytes (S100A10) and prorepair microglia/monocytes (CD163) was very low in the corpus callosum of mice consuming a regular diet, with slightly elevated levels of S100A10 detected in PAR1 ${ }^{-/-}$mice. Levels of S100A10 and CD163, remained low across genotypes after 6 weeks of CPZ consumption (data not shown), but increased after 3 weeks of induced repair, with levels of CD163 being significantly higher in PAR1 ${ }^{-I-}$ mice. Each bar represents the mean value from $n=4-5$ animals for each genotype and time point. Asterisks represent significant differences with ${ }^{*} p<0.05 ;{ }^{* *} p<0.01$; ${ }^{* * *} p \leq 0.001$, Student's $t$ test. Scale bar, $50 \mu \mathrm{m}$.

tioned media from astrocytes derived from PAR1 knock-out mice, showed increases in Olig2 ${ }^{+}$counts (Fig. 8b: $\mathrm{df}=8, t=$ $-3.20, p=0.01$, Student's $t$ test; Fig. $9 a$ : one-way ANOVA, $\mathrm{df}=$ $3, F=20.36, p=0.004, \mathrm{NK}$ ) and in the abundance of PLP per cell (Fig. 9a: one-way ANOVA, $\mathrm{df}=3, F=2.99, p=0.01$, NK; Fig. $8 b: \mathrm{df}=8, t=-2.66, p=0.02$, Student's $t$ test). These results suggest that PAR1 loss of function in astrocytes generates promyelinating signal(s) that contribute to enhancements in the myelin generating potential of OPCs.

To further investigate the potential regulatory role of astrocyte PAR1, we examined factors related to astrocyte proinflammatory or prorepair properties in RNA isolated from wild-type or PAR1 knock-out astrocyte cultures using quantitative real time PCR (Fig. 8c). First, we determined the expression of GFAP, aldehyde dehydrogenase 1 family member L1 (Aldh1L1), vimentin (VIM) and signal transducer and activator of transcription 3 (STAT3) to

\section{$\leftarrow$}

(Figure legend continued.) Elevations in Iba1 as a result of 6 weeks of $\mathrm{CPZ}$ consumption were attenuated in PAR1 ${ }^{-l-}$ mice $(p=0.02)$. Staining for markers of prorepair astrocytes (S100A10) and prorepair microglia/monocytes (CD163) were very low in the CPZ treated corpus callosum at all time points in PAR1 ${ }^{-1-}$ mice, but elevations in CD163 were readily detectable using immunoperoxidase methods 3 weeks after returning mice to regular chow to induce repair in PAR1 ${ }^{-1-}$ mice (Fig. 7b). Asterisks represent significant differences with ${ }^{*} p<0.05$; ${ }^{* *} p<0.01 ;{ }^{* *} p \leq 0.001$, Student's $t$ test, $n=4$ for each genotype. Scale bar, $100 \mu \mathrm{m}$. gauge astrocyte reactivity and complemented these results with quantification of proinflammatory ( $\mathrm{H}-2$ class I histocompatibility antigen, H2D1) or prorepair (S100A10, CD14, cardiotrophin like cytokine factor 1 (Clcf1)) signatures within the astrocyte transcriptome. Compared with wild-type astrocytes, those lacking PAR1 showed 2.4-fold higher levels of GFAP ( $\mathrm{df}=4, t=$ $-7.69, p=0.002$ ) and 1.2-fold higher levels of VIM RNA expression $(\mathrm{df}=4, t=-2.97, p=0.04)$. PAR1 knock-out astrocytes also showed lower levels of $\mathrm{H} 2 \mathrm{D} 1(\mathrm{df}=4, t=6.02, p=0.004)$ and higher levels of S100A10 (df $=4, t=-6.94, p=0.002)$ and $\mathrm{CD} 14(\mathrm{df}=4, t=-6.67, p=0.003) \mathrm{RNA}$ expression compared with wild-type astrocytes (Student's $t$ test). PAR1 knock-out astrocyte cultures expressed lower levels of the proinflammatory cytokine interleukin $1 \beta(\mathrm{IL}-1 \beta)(\mathrm{df}=4, t=3.14, p=0.03)$, with expression of tumor necrosis factor (TNF) and interleukin 6 (IL-6) unchanged. Notably, transforming growth factor $\beta$ (TGF $\beta$ ), a cytokine characterized as prorepair was elevated by 1.2-fold in astrocytes lacking the PAR1 gene ( $\mathrm{df}=3, t=-6.62$, $p=0.007$, Student's $t$ test). Astrocytes with genetic PAR1 loss of function also showed increases in two promyelinating growth factors BDNF (1.3-fold, $\mathrm{df}=4, t=-2.97, p=0.04)$ and insulin like growth factor-1 (IGF-1) (1.4-fold, $\mathrm{df}=4, t=-5.93, p=$ 0.001 , Student's $t$ test), while increases in leukemia inhibitory factor (LIF1) and thrombospondin 1 (TSP1) did not reach statistical significance. These results demonstrate that PAR1 gene 
a

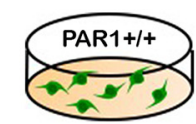

Untreated

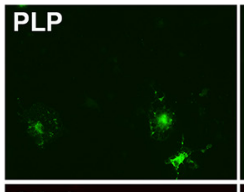

Olig2
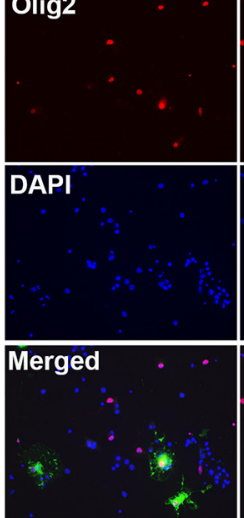

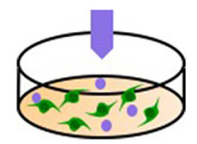

Vorapaxar

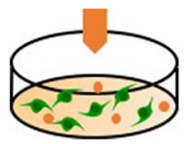

BDNF

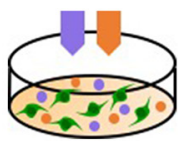

Vorapaxar+BDNF
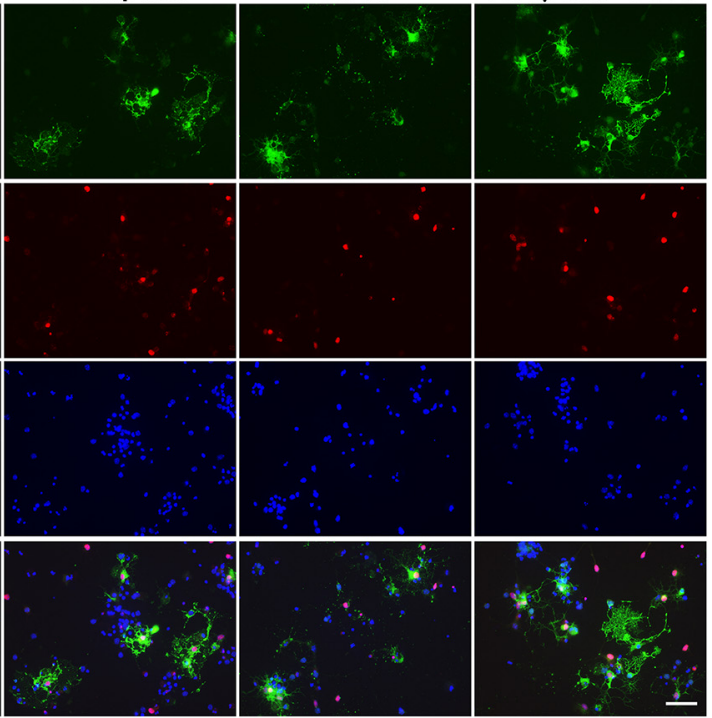

b

(+) Vorapaxar (18 h)

(+) PAR1+/+ OPCs

Culture for $72 \mathrm{~h}$
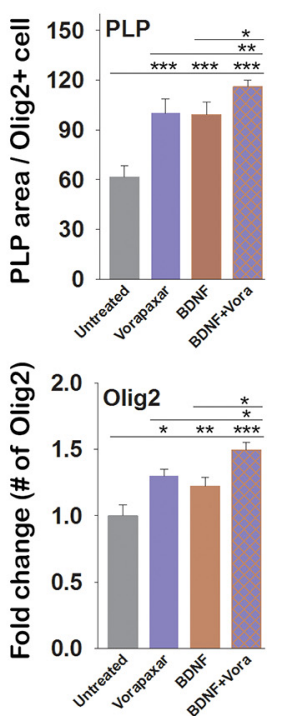
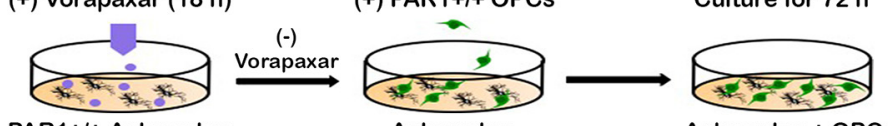

PAR1+/+ Astrocytes

Astrocytes

Astrocytes + OPCs
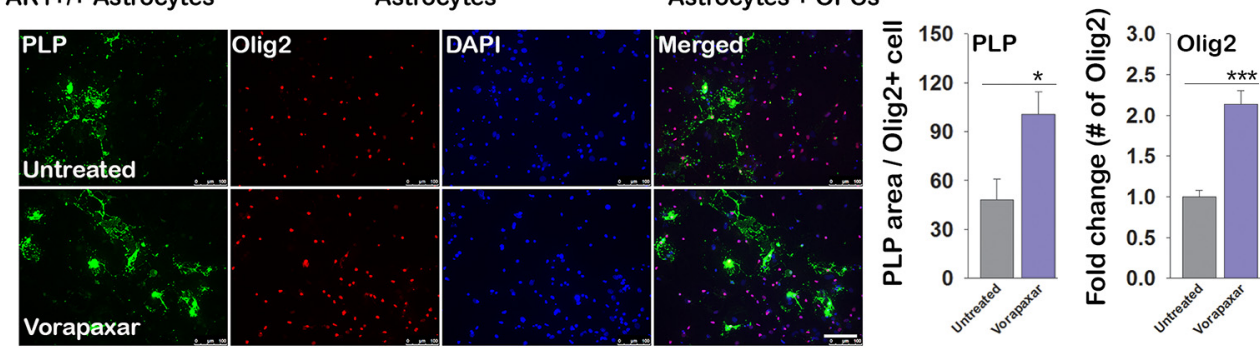

C

\section{Astrocyte Markers}

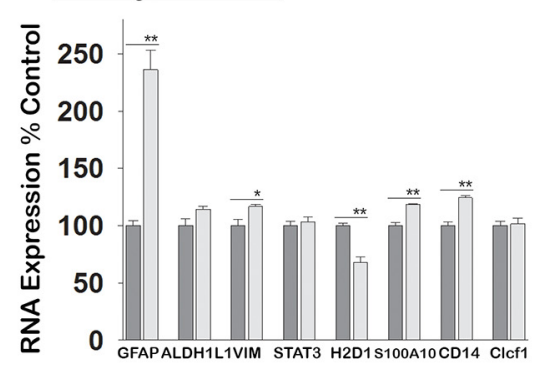

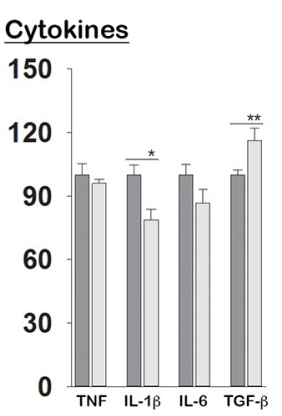

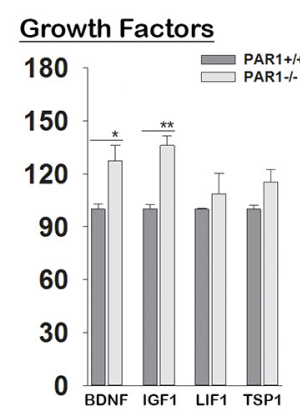

Figure 8. PAR1 loss of function enhances myelin production by oligodendrocyte progenitor cells by possible direct and indirect mechanisms. $\boldsymbol{a}$, Wild-type cortical oligodendrocyte progenitor cells (OPCs) were treated for $24 \mathrm{~h}$ with the PAR1 inhibitor vorapaxar (100 nM), or suboptimal levels of BDNF (1 ng/ml) alone, or in combination. Images and associated histograms show colocalization of immunofluorescence for PLP and 0lig2 in each condition after $72 \mathrm{~h}$. Inhibition of OPC PAR1 alone increased the relative area of PLP per 0lig2 ${ }^{+}$cell and the number of 0 lig $2^{+}$cells to a similar extent as $1 \mathrm{ng} / \mathrm{ml} \mathrm{BDNF}$, with additive effects seen in combination. $\boldsymbol{b}$, Wild-type cortical astrocytes treated with the PAR1 inhibitor vorapaxar (100 nM) for $18 \mathrm{~h}$, then cultured with PAR1 ${ }^{+/+} \mathrm{OPCs}$ for $72 \mathrm{~h}$ enhanced the number of 0 lig $2^{+}$cells and the area of PLP stained per Olig2 ${ }^{+}$cell. c, Histograms showing differences in expression of astrocyte related markers, including cytokines and growth factors in RNA isolated from PAR1 ${ }^{+/+}$or PAR1 ${ }^{-1-}$ cortical astrocytes and quantified using real time qPCR. PAR1 ${ }^{-/-}$ astrocytes showed increases in expression of general astrocyte markers GFAP and vimentin (VIM), increases in astrocyte markers considered prorepair (S100A10 and CD14) and decreases in H2D1 an astrocyte marker considered proinflammatory. The proinflammatory cytokine IL- $1 \beta$ was decreased in PAR $1^{-1-}$ astrocytes, while TGF- $\beta$, considered to have a more anti-inflammatory role, was increased. Growth factors playing roles in oligodendrocyte survival and differentiation (BDNF and IGF1) were both increased in PAR1 ${ }^{-1-}$ astrocytes. Statistical evaluation in $\boldsymbol{a}$ was done by one-way ANOVA followed by NK post hoc test and in $\boldsymbol{b}$ and $\boldsymbol{c}$ by Student's $t$ test. Asterisks represent significant differences with ${ }^{*} p<0.05$; ${ }^{* *} p<$ $0.01 ;{ }^{* * *} p \leq 0.001$. Scale bars in $\boldsymbol{a}$ and $\boldsymbol{b}$ indicate $100 \mu \mathrm{m}$. 
a

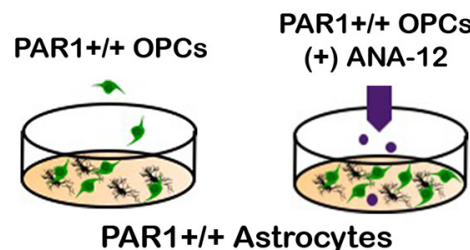

PAR1+/+ Astrocytes

PAR1+/+

Untreated ANA-12(10)
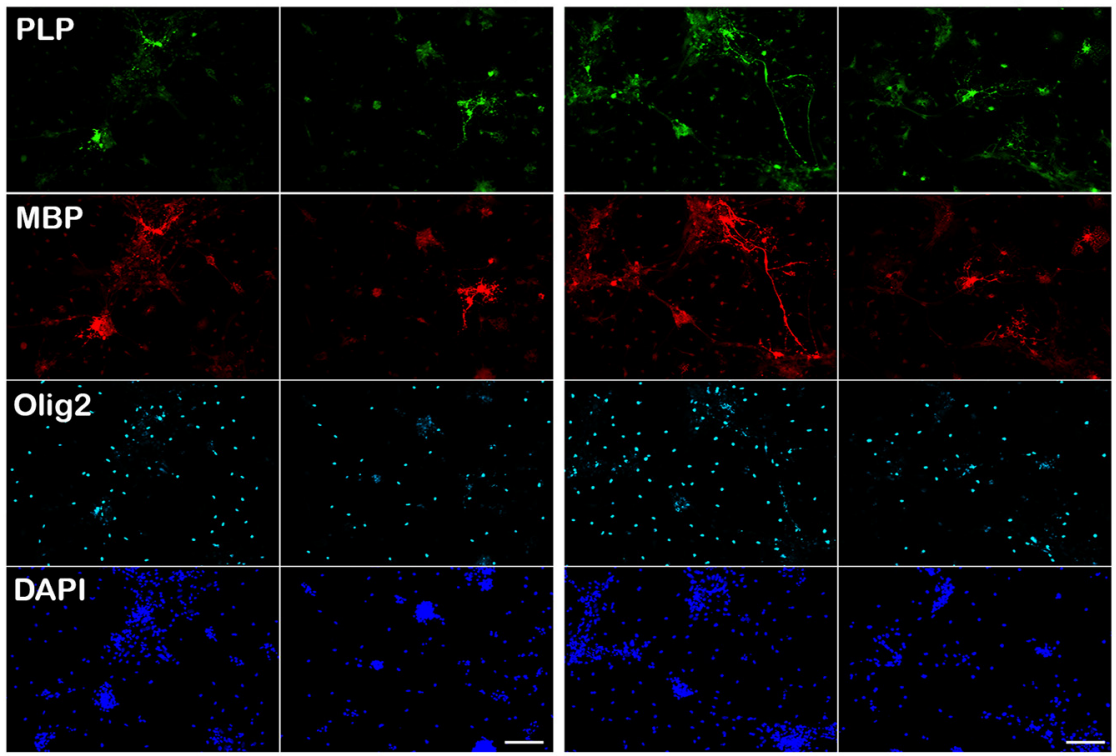

b
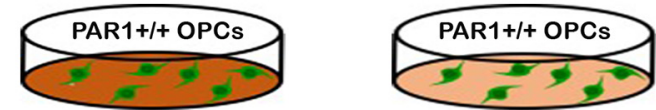

PAR1+/+ ACM

PAR1-I- ACM
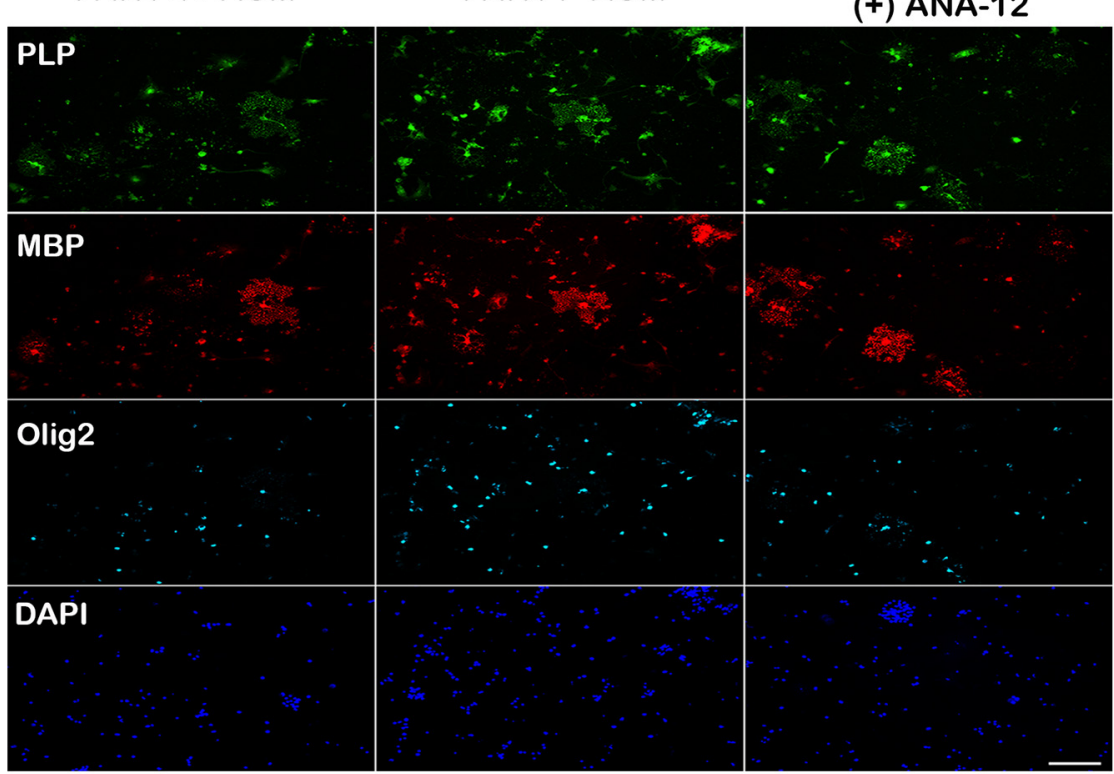

PAR1+/+ OPCs

(+) ANA-12
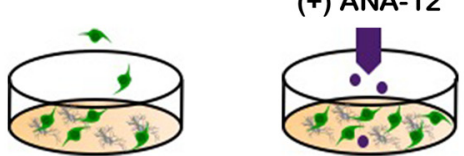

PAR1-/- Astrocytes

PAR1-1-

Untreated ANA-12(10)
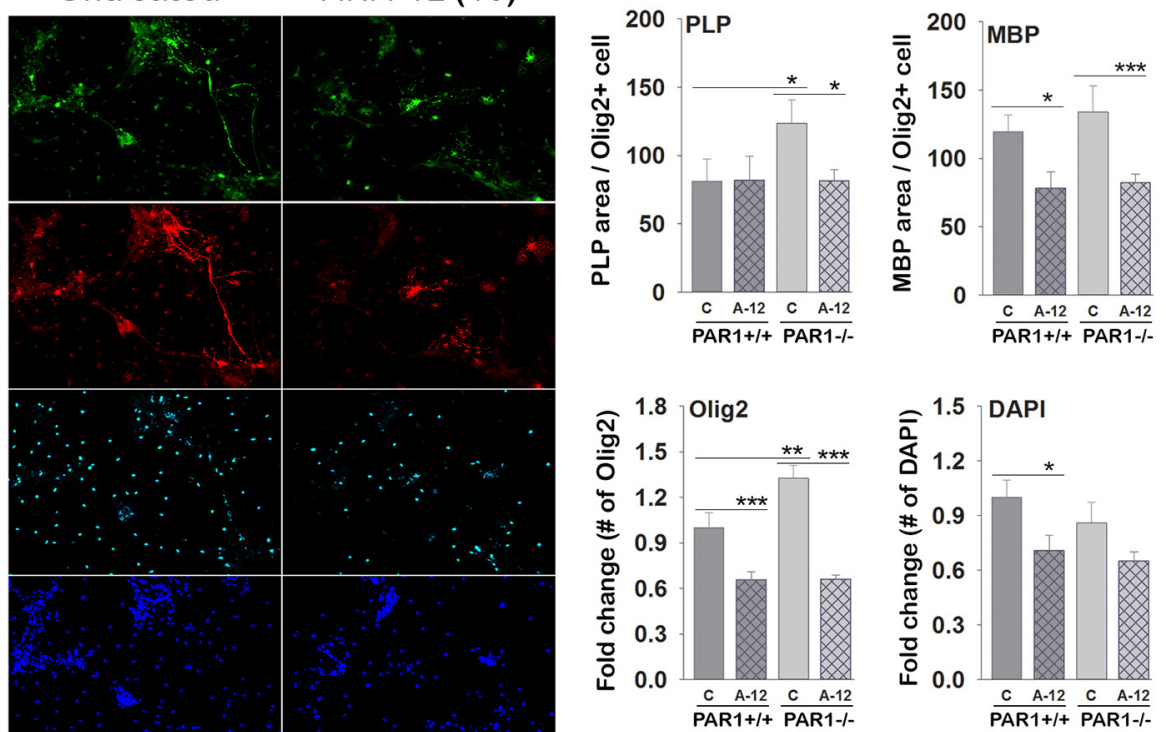
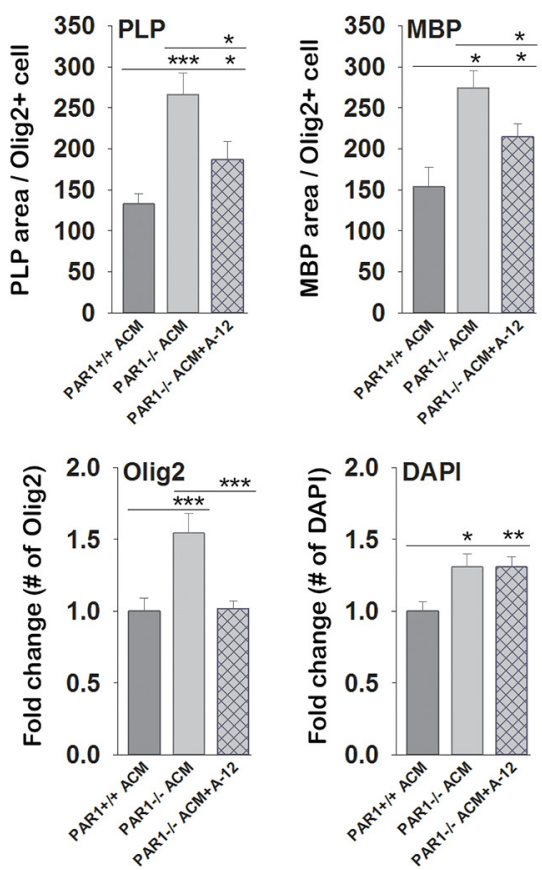

Figure 9. The promyelinating effects of astrocytes with PAR1 loss of function depends on oligodendrocyte progenitor cell TrkB. $\boldsymbol{a}$, Images showing immunofluorescence for PLP (green), MBP (red), and Olig2 (cyan) in cocultures of PAR1 ${ }^{+/+}$OPCs grown on a monolayer of either PAR1 ${ }^{+/+}$or PAR1 ${ }^{-/-}$astrocytes in the presence or absence of the TrkB inhibitor ANA-12. Histograms with corresponding quantification show that PAR1 ${ }^{-/-}$astrocytes support increases in the abundance of 0 lig2 ${ }^{+}$cells and expression of both PLP and MBP per 0lig2 ${ }^{+}$cell, and that these beneficial effects are lost when ANA-12 is included in the media. $\boldsymbol{b}$, The $10-100 \mathrm{kDa}$ fraction of astrocyte conditioned media taken from PAR ${ }^{-1-}$ astrocytes also promotes increases in the number of 0 lig ${ }^{+}$ cells and in the amount of PLP and MBP expression per cell, and again, beneficial effects were blocked when ANA-12 was included in the media. Asterisks represent significant differences with ${ }^{*} p<$ $0.05 ;{ }^{* *} p<0.01 ;{ }^{* * *} p \leq 0.001$, one-way ANOVA followed by NK post hoc test. Scale bars in $\boldsymbol{a}$ and $\boldsymbol{b}$ indicate $100 \mu \mathrm{m}$. 
knock-out significantly improves the neural repair profile of astrocytes, including increases in the expression of BDNF, IGF-1 and TGF $\beta$, which may influence OPC differentiation through paracrine signaling mechanisms.

Given the elevated levels of BDNF RNA expressed by PAR1 knock-out astrocytes in vitro (Fig. $8 \mathrm{c}$ ) and the additive promyelinating effects of BDNF applied jointly with PAR1 inhibition (Fig. $8 a$ ), we sought to investigate the potential role of BDNF-derived from PAR1 knock-out astrocytes in conferring promyelinating effects (Fig. 9). First, we found that cocultures of wild-type OPCs cultured with PAR1 knock-out astrocytes expressed higher levels of Olig2 and PLP compared with those grown in coculture with wild-type astrocytes (Fig. 9a, one-way ANOVA, Olig2; df = 3, $F=20.36, p=0.004, \mathrm{PLP} ; \mathrm{df}=3, F=2.99, p=0.01, \mathrm{NK})$. Moreover, the promyelinating effects of PAR1 knock-out astrocytes were blocked by inclusion of an antagonist of the highaffinity receptor for BDNF, TrkB (ANA-12) in the culture media (one-way ANOVA, Olig2; $\mathrm{df}=3, F=20.36, p<0.001$, PLP; $\mathrm{df}=$ $3, F=4.8, p=0.01, \mathrm{MBP} ; \mathrm{df}=3, F=9.89, p=0.003, \mathrm{NK})$. Next, we demonstrated that TrkB inhibition also reduces the promyelinating effects of PAR1 knock-out astrocyte conditioned media (Fig. 9b, one-way ANOVA, Olig2; df $=2, F=9.24, p<0.001$, PLP; $\mathrm{df}=2, F=10.52, p<0.001, \mathrm{MBP} ; \mathrm{df}=2, F=8.77, p<$ $0.001, \mathrm{NK})$. These results suggest that increases in astrocyte BDNF expression generated by PAR1 loss of function are positioned to contribute to the promyelinating effects we observed in vitro.

\section{Discussion}

PAR1 is a protease activated G-protein coupled receptor densely expressed in the CNS, however to date there exists limited information regarding its physiological roles in health and disease. Given studies demonstrating PAR1 gain-of-function reduces myelin proteins (Burda et al., 2013), whereas PAR1 loss of function accelerates myelin development (Yoon et al., 2015), we set out to test the hypothesis that PAR1 inhibition is sufficient to promote myelin regeneration and to provide insights into its actions across glial compartments. Findings demonstrate that blocking PAR1 improves CNS myelin regeneration by a mechanism that possibly involves both OPC-direct and indirect effects that include astrocyte-OPC trophic coupling. These findings underscore the fundamental roles of PAR1 in myelin biology and potential to integrate with powerful growth factor systems, such as BDNF to improve myelin regeneration in the adult CNS.

\section{Blocking PAR1 promotes remyelination in multiple models}

PAR1 knock-out resulted in improved recovery of OPC and mature oligodendrocyte numbers, in addition to greater axon remyelination in the lysolecithin model. We confirmed these findings in a second model involving chronic demyelination where mature oligodendrocytes are killed by cuprizone toxicity. Even in this chronic myelin injury model, improvements in OPCs and mature oligodendrocytes, signs of axonal protection and preserved motor function were observed PAR1 knock-outs. Because the current study focused on male mice, future studies are warranted confirm findings in females. Increases in myelin regeneration with PAR1 knock-out compare favorably to remyelination drug candidates, including LINGO1 (Mi et al., 2005, 2008), rHIgM22 (Mullin et al., 2017) and Clemastine (Deshmukh et al., 2013; Mei et al., 2016; Eleuteri et al., 2017). Taken with prior studies demonstrating PAR1 knock-outs show accelerated myelin development (Yoon et al., 2015), recognition of the role of PAR1 in myelin regeneration highlight it as an important regulator of myelin biology in health and disease.

\section{Blocking PAR1 promotes remyelination through possible OPC-direct mechanisms}

Although the potential influence of a developmental benefit, including differences in myelin aging as a result of PAR1 knock-out cannot be fully ruled out (Yoon et al., 2015; Choi et al., 2018), beneficial effects on the process of remyelination are suggested by several observations. First, whereas lysolecithin and cuprizone caused equal loss of mature myelinating cells and MBP across genotypes, signs of myelin replacement, including increased numbers of mature oligodendrocytes were greater in PAR1 knock-outs. Moreover, increases in oligodendrocyte proliferation during myelin regeneration after Cuprizone were augmented in PAR1 knock-out mice. In vitro studies are also consistent with possible direct and acute benefits of PAR1 loss of function on myelinating cells with a PAR1 inhibitor, vorapaxar, increasing PLP abundance. Considering prior studies demonstrating excess PAR1 activation suppresses myelin (Burda et al., 2013), we suggest OPC-PAR1 serves as a molecular switch regulating myelination. Supporting a model where turning OFF PAR1 in OPCs promotes myelin production (Fig. 10), PAR1 is expressed by PDGFR ${ }^{+}$OPCs (Yoon et al., 2015) and is among the most downregulated genes upon differentiation (Cahoy et al., 2008). Since sites of neurological injury can be enriched in PAR1 activating enzymes such as thrombin, kallikrein 6, plasmin and MMP-1 (Scarisbrick, 2008), PAR1 represents a target to improve myelin regeneration. Results here provide rationale for efforts to demonstrate whether blocking PAR1 after demyelination also enhances signs of myelin regeneration.

\section{Blocking PAR1 promotes remyelination through possible OPC-indirect mechanisms}

While the current and prior findings support possible direct regulatory roles for PAR1 in OPC proliferation and differentiation, PAR1-OPC-extrinsic promyelinating signals involving astrocytes (Nash et al., 2011; Hibbits et al., 2012; Skripuletz et al., 2013; Hammond et al., 2014) and microglia (Miron et al., 2013; Lampron et al., 2015; Lloyd et al., 2019) are likely. For example, MS-relevant proteases such as thrombin and kallikrein 6 evoke PAR1-dependent astrocyte $\mathrm{Ca}^{2+}$ flux, MAPK signaling, and IL-6 secretion (Vandell et al., 2008; Scarisbrick et al., 2012; Radulovic et al., 2016; Yoon et al., 2018). PAR1 differentially regulates astrocyte glutamate release and reuptake (Sweeney et al., 2017) and astrocyte proliferation (Nicole et al., 2005). In microglia, PAR1 potentiates CD40 ligand-induced TNF expression (Suo et al., 2002). Coupled to these findings, PAR1 knock-outs show attenuated astroglial and microglial responses across neural injury models, including cortical stab wound (Nicole et al., 2005), traumatic SCI (Radulovic et al., 2016), and ischemia (Rajput et al., 2014). Here, PAR1 knock-outs showed improved remyelination and a skewing of astrocyte and microglial signatures toward a prorepair phenotype, with unique changes depending on the acute or chronic nature of myelin injury.

Improved remyelination in PAR1 knock-outs in the lysolecithin model were associated with increased astrocyte S100A10. S100A10 is a calcium-binding protein implicated in cell cycle and differentiation and with neurotrophic support and modulation of inflammatory astrocytes (Liddelow et al., 2017). By contrast, in the cuprizone model of chronic demyelination, S100A10 was not strongly induced, but rather improved remyelination was associated with reductions in the astrocyte proinflammatory marker 


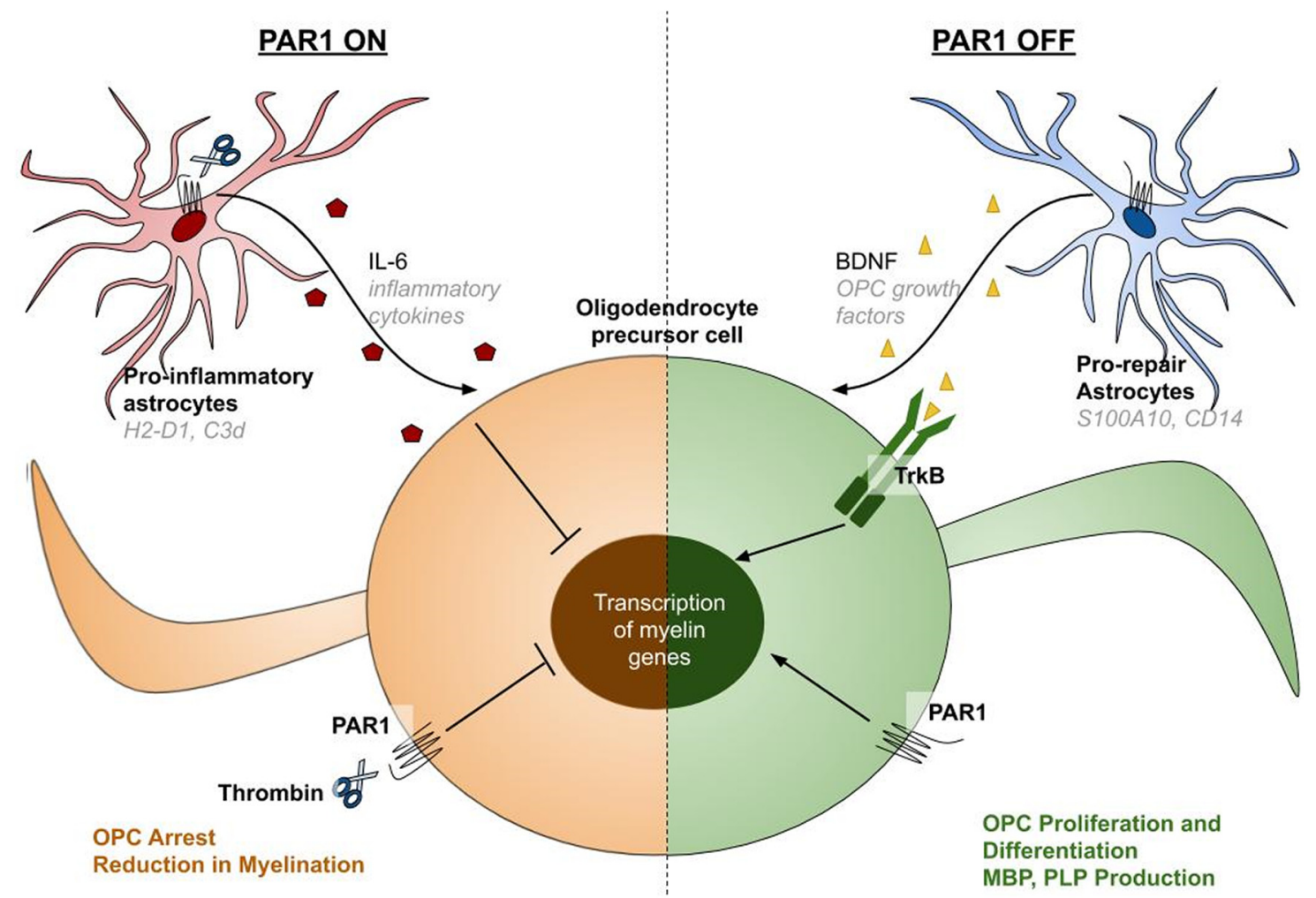

Figure 10. Working model of repair processes influenced by PAR1 knock-out. In this working model, PAR1 serves as a sensor of the protease microenvironment with protease-mediated PAR-activation (SWITCH ON, as would occur in the context of neuroinflammation) resulting in a suppression of myelin production (OPC arrest), while PAR-inhibition (SWITCH OFF) is myelination permissive. Findings suggest the PAR1 molecular SWITCH can exert control on oligodendrocyte differentiation both by possible direct effects at the level of OPCs and indirect effects involving astrocytes. Activation of OPC PAR1 suppresses transcription of myelin genes resulting in reduced myelin production, while switching OFF OPC PAR1 promotes OPC proliferation and differentiation, including MBP and PLP production. Astrocytes also express PAR1 and indeed upregulate this receptor in the context of demyelination. Astrocytes with PAR1 activation secrete proinflammatory oligodendrocyte damaging cytokines, such as IL-6 and express $\mathrm{H} 2 \mathrm{D} 1$ a signature of a proinflammatory phenotype. By contrast, switching OFF astrocyte PAR1 leads to expression of prorepair astrocyte markers, such as S100A10 and increased production of promyelinating growth factors such as BDNF. BDNF in turn drives oligodendrocyte production and differentiation through TrkB activation. This model is consistent with results in the current study that demonstrate switching OFF PAR1 leads to improvements in myelin regeneration in the adult brain and spinal cord.

Complement 3 (C3d). C3d, identified as directing proinflammatory properties, is involved in innate immunity by complement activation and is linked to synaptic pruning and neurodegeneration upon upregulation (Liddelow et al., 2017; Clarke et al., 2018; Yun et al., 2018).

Consistent with findings demonstrating higher GFAP in the spinal cord of PAR1 knock-outs compared with wild-type mice (Radulovic et al., 2016), we observed elevations in GFAP in the corpus callosum of PAR1 knock-outs at baseline, before demyelination. GFAP is an intermediate filament protein commonly used to identify astrocyte reactivity, but is not necessarily associated with proinflammatory or prorepair responses. Mirroring increases in S100A10 during acute lysolecithin remyelination in PAR1 knock-outs, GFAP levels were also increased. Paralleling decreases in astrocyte C3d in PAR1 knock-outs during remyelination in the chronic cuprizone model, GFAP levels were also reduced. While additional study regarding the role(s) of PAR1 in regulating astrocyte proinflammatory and prorepair signatures in vivo awaits experiments using conditional knock-outs, the current findings suggest global PAR1 blockade is a powerful method to skew astrocyte signatures toward a phenotype favoring myelin regeneration.

Microglia and monocytes play significant roles in demyelination and the success of myelin regeneration (Miron et al., 2013; Lloyd et al., 2019; Wies Mancini et al., 2019), and indeed direct proinflammatory and prorepair astrocyte properties (Skripuletz et al., 2013; Liddelow et al., 2017). After 6 weeks of cuprizone demyelination, the robust increases in microglial Ibal were reduced in PAR1 knock-outs. Moreover, there were reductions in CD68, a marker of reactive microglial/monocytes at 6 and 3 weeks of repair, while CD163, a prorepair marker, was increased. Notably, previous cuprizone studies demonstrated that prorepair microglia secrete factors that improve OPC survival, proliferation, and migration (Miron et al., 2013). The same microglial markers did not differ between genotypes during lysolecithin remyelination, although improvements in repair persisted. Altogether, these findings suggest PAR1 loss of function in the context of chronic cuprizone demyelination skews microglial responses toward a phenotype that favors myelin regeneration and further investigation of direct role(s) in repair, or in directing the properties of astrocytes, using a larger number of markers and conditional PAR1 knock-out mice, are future directions.

\section{Blocking PAR1 enhances myelin production in part through a BDNF-TrkB-dependent mechanism}

Given the skewing of astrocyte responses in PAR1 knock-out mice toward a prorepair phenotype across models, we next investigated whether PAR1 loss of function specifically in astrocyte cultures is sufficient to promote parallel changes and any biological influence toward OPCs. Parallel to results in vivo (Radulovic et al., 2016), higher levels of GFAP were expressed by PAR1 knock-out astrocytes in vitro. Underscoring that GFAP is not necessarily proinflammatory or prorepair, PAR1 knock-out astrocyte cultures concomitantly showed decreased H2D1 and 
IL-1 $\beta$ expression, each a proinflammatory signature. Complementing these findings, PAR1 knock-out astrocytes also showed coordinate increases in S100A10 and CD14 prorepair signatures and TGF- $\beta$, a cytokine with immune regulatory with prorepair effects (Fok-Seang et al., 1998; Hamaguchi et al., 2019). In addition to skewed cytokine responses, astrocyte PAR1 loss of function was also sufficient to increase expression of BDNF and IGF1, two growth factors with well studied promyelinating properties (Min et al., 2012; Fulmer et al., 2014).

Using astrocyte-OPC cocultures or astrocyte-conditioned media applied to OPCs, we next determined that pharmacologic or genetic astrocyte PAR1 loss of function was sufficient to increase PLP and MBP. The promyelin effects of astrocyte PAR1 loss-of function were reduced when an inhibitor of the highaffinity BDNF receptor, TrkB, was included in astrocyte-OPC cocultures, or along with PAR1 knock-out astrocyte conditioned media. These findings suggest that increases in BDNF RNA expression in PAR1-knock-out astrocytes may contribute to promyelinating effects. Although these findings do not exclude roles of PAR1-regulated cytokines or other growth factors, including IGF1 (Mason et al., 2003; Zeger et al., 2007), BDNF is well recognized to promote CNS myelination and myelin regeneration (VonDran et al., 2011; Wong et al., 2013; Fulmer et al., 2014; Ramos-Cejudo et al., 2015; Fletcher et al., 2018). Findings that astrocyte PAR1 loss of function increases BDNF expression and that OPC PAR1 loss of function augments BDNF's promyelinating effects are of particular significance since they suggest that blocking PAR1 may improve myelin production by augmenting the efficacy of available growth factors often depleted at sites of neural injury.

\section{References}

Armstrong RA (2014) When to use the bonferroni correction. Ophthalmic Physiol Opt 34:502-508.

Bielecki B, Mattern C, Ghoumari AM, Javaid S, Smietanka K, Abi Ghanem C, Mhaouty-Kodja S, Ghandour MS, Baulieu EE, Franklin RJ, Schumacher M, Traiffort E (2016) Unexpected central role of the androgen receptor in the spontaneous regeneration of myelin. Proc Natl Acad Sci U S A 113:14829-14834.

Bothwell M (2017) Mechanisms and medicines for remyelination. Annu Rev Med 68:431-443.

Bouhrara M, Reiter DA, Bergeron CM, Zukley LM, Ferrucci L, Resnick SM, Spencer RG (2018) Evidence of demyelination in mild cognitive impairment and dementia using a direct and specific magnetic resonance imaging measure of myelin content. Alzheimers Dement 14:998-1004.

Burda JE, Radulovic M, Yoon H, Scarisbrick IA (2013) Critical role for PAR1 in kallikrein 6-mediated oligodendrogliopathy. Glia 61:1456-1470.

Cahoy JD, Emery B, Kaushal A, Foo LC, Zamanian JL, Christopherson KS, Xing Y, Lubischer JL, Krieg PA, Krupenko SA, Thompson WJ, Barres BA (2008) A transcriptome database for astrocytes, neurons, and oligodendrocytes: a new resource for understanding brain development and function. J Neurosci 28:264-278.

Choi CI, Yoon H, Drucker KL, Langley MR, Kleppe L, Scarisbrick IA (2018) The thrombin receptor restricts subventricular zone neural stem cell expansion and differentiation. Sci Rep 8:9360.

Clarke LE, Liddelow SA, Chakraborty C, Munch AE, Heiman M, Barres BA (2018) Normal aging induces A1-like astrocyte reactivity. Proc Natl Acad Sci U S A 115:E1896-E1905.

Deshmukh VA, Tardif V, Lyssiotis CA, Green CC, Kerman B, Kim HJ, Padmanabhan K, Swoboda JG, Ahmad I, Kondo T, Gage FH, Theofilopoulos AN, Lawson BR, Schultz PG, Lairson LL (2013) A regenerative approach to the treatment of multiple sclerosis. Nature 502:327-332.

Dugas JC, Emery B (2013) Purification and culture of oligodendrocyte lineage cells. Cold Spring Harbor Protoc 2013:810-814.

Eleuteri C, Olla S, Veroni C, Umeton R, Mechelli R, Romano S, Buscarinu MC, Ferrari F, Calo G, Ristori G, Salvetti M, Agresti C (2017) A staged screening of registered drugs highlights remyelinating drug candidates for clinical trials. Sci Rep 7:45780.
Fletcher JL, Wood RJ, Nguyen J, Norman EML, Jun CMK, Prawdiuk AR, Biemond M, Nguyen HTH, Northfield SE, Hughes RA, Gonsalvez DG, Xiao J, Murray SS (2018) Targeting TrkB with a brain-derived neurotrophic factor mimetic promotes myelin repair in the brain. J Neurosci 38:7088-7099.

Fok-Seang J, DiProspero NA, Meiners S, Muir E, Fawcett JW (1998) Cytokine-induced changes in the ability of astrocytes to support migration of oligodendrocyte precursors and axon growth. Eur J Neurosci 10:2400-2415.

Fulmer CG, VonDran MW, Stillman AA, Huang Y, Hempstead BL, Dreyfus CF (2014) Astrocyte-derived BDNF supports myelin protein synthesis after cuprizone-induced demyelination. J Neurosci 34:8186-8196.

Hamaguchi M, Muramatsu R, Fujimura H, Mochizuki H, Kataoka H, Yamashita T (2019) Circulating transforming growth factor-betal facilitates remyelination in the adult central nervous system. Elife 8:e41869.

Hammond TR, Gadea A, Dupree J, Kerninon C, Nait-Oumesmar B, Aguirre A, Gallo V (2014) Astrocyte-derived endothelin-1 inhibits remyelination through notch activation. Neuron 81:588-602.

Hibbits N, Yoshino J, Le TQ, Armstrong RC (2012) Astrogliosis during acute and chronic cuprizone demyelination and implications for remyelination. ASN Neuro 4:393-408.

Karamita M, Barnum C, Mobius W, Tansey MG, Szymkowski DE, Lassmann H, Probert L (2017) Therapeutic inhibition of soluble brain TNF promotes remyelination by increasing myelin phagocytosis by microglia. JCI Insight 2:e87455.

Lampron A, Larochelle A, Laflamme N, Prefontaine P, Plante MM, Sanchez MG, Yong VW, Stys PK, Tremblay ME, Rivest S (2015) Inefficient clearance of myelin debris by microglia impairs remyelinating processes. J Exp Med 212:481-495.

Liddelow SA, Guttenplan KA, Clarke LE, Bennett FC, Bohlen CJ, Schirmer L, Bennett ML, Münch AE, Chung W-S, Peterson TC, Wilton DK, Frouin A, Napier BA, Panicker N, Kumar M, Buckwalter MS, Rowitch DH, Dawson VL, Dawson TM, Stevens B, et al. (2017) Neurotoxic reactive astrocytes are induced by activated microglia. Nature 541:481-487.

Lloyd AF, Davies CL, Holloway RK, Labrak Y, Ireland G, Carradori D, Dillenburg A, Borger E, Soong D, Richardson JC, Kuhlmann T, Williams A, Pollard JW, des Rieux A, Priller J, Miron VE (2019) Central nervous system regeneration is driven by microglia necroptosis and repopulation. Nat Neurosci 22:1046-1052.

Mason JL, Xuan S, Dragatsis I, Efstratiadis A, Goldman JE (2003) Insulinlike growth factor (IGF) signaling through type 1 IGF receptor plays an important role in remyelination. J Neurosci 23:7710-7718.

Matsushima GK, Morell P (2001) The neurotoxicant, cuprizone, as a model to study demyelination and remyelination in the central nervous system. Brain Pathol 11:107-116.

Mei F, Lehmann-Horn K, Shen YA, Rankin KA, Stebbins KJ, Lorrain DS, Pekarek K, S AS, Xiao L, Teuscher C, von Budingen HC, Wess J, Lawrence JJ, Green AJ, Fancy SP, Zamvil SS, Chan JR (2016) Accelerated remyelination during inflammatory demyelination prevents axonal loss and improves functional recovery. Elife 5:e18246.

Mi S, Miller RH, Lee X, Scott ML, Shulag-Morskaya S, Shao Z, Chang J, Thill G, Levesque M, Zhang M, Hession C, Sah D, Trapp B, He Z, Jung V, McCoy JM, Pepinsky RB (2005) LINGO-1 negatively regulates myelination by oligodendrocytes. Nat Neurosci 8:745-751.

Mi S, Sandrock A, Miller RH (2008) LINGO-1 and its role in CNS repair. Int J Biochem Cell Biol 40:1971-1978.

Min J, Singh S, Fitzgerald-Bocarsly P, Wood TL (2012) Insulin-like growth factor I regulates G2/M progression through mammalian target of rapamycin signaling in oligodendrocyte progenitors. Glia 60:1684-1695.

Miron VE, Boyd A, Zhao JW, Yuen TJ, Ruckh JM, Shadrach JL, van Wijngaarden $\mathrm{P}$, Wagers AJ, Williams A, Franklin RJ, ffrench-Constant C (2013) M2 microglia and macrophages drive oligodendrocyte differentiation during CNS remyelination. Nat Neurosci 16:1211-1218.

Mullin AP, Cui C, Wang Y, Wang J, Troy E, Caggiano AO, Parry TJ, Colburn RW, Pavlopoulos E (2017) rHIgM22 enhances remyelination in the brain of the cuprizone mouse model of demyelination. Neurobiol Dis 105:142-155.

Nash B, Thomson CE, Linington C, Arthur AT, McClure JD, McBride MW, Barnett SC (2011) Functional duality of astrocytes in myelination. J Neurosci 31:13028-13038.

Nasrabady SE, Rizvi B, Goldman JE, Brickman AM (2018) White matter 
changes in Alzheimer's disease: a focus on myelin and oligodendrocytes. Acta Neuropathol Commun 6:22.

Nicole O, Goldshmidt A, Hamill CE, Sorensen SD, Sastre A, Lyuboslavsky P, Hepler JR, McKeon RJ, Traynelis SF (2005) Activation of proteaseactivated receptor-1 triggers astrogliosis after brain injury. J Neurosci 25:4319-4329.

Patrikios P, Stadelmann C, Kutzelnigg A, Rauschka H, Schmidbauer M, Laursen H, Sorensen PS, Bruck W, Lucchinetti C, Lassmann H (2006) Remyelination is extensive in a subset of multiple sclerosis patients. Brain 129:3165-3172.

Radulovic M, Yoon H, Wu J, Mustafa K, Scarisbrick IA (2016) Targeting the thrombin receptor modulates inflammation and astrogliosis to improve recovery after spinal cord injury. Neurobiol Dis 93:226-242.

Rajput PS, Lyden PD, Chen B, Lamb JA, Pereira B, Lamb A, Zhao L, Lei IF, Bai J (2014) Protease activated receptor-1 mediates cytotoxicity during ischemia using in vivo and in vitro models. Neuroscience 281C:229-240.

Ramos-Cejudo J, Gutierrez-Fernandez M, Otero-Ortega L, Rodriguez-Frutos B, Fuentes B, Vallejo-Cremades MT, Hernanz TN, Cerdan S, DiezTejedor E (2015) Brain-derived neurotrophic factor administration mediated oligodendrocyte differentiation and myelin formation in subcortical ischemic stroke. Strokem46:221-228.

Scarisbrick IA (2008) The multiple sclerosis degradome: enzymatic cascades in development and progression of central nervous system inflammatory disease. Curr Top Microbiol Immunol 318:133-175.

Scarisbrick IA, Blaber SI, Lucchinetti CF, Genain CP, Blaber M, Rodriguez M (2002) Activity of a newly identified serine protease in CNS demyelination. Brain 125:1283-1296.

Scarisbrick IA, Radulovic M, Burda JE, Larson N, Blaber SI, Giannini C, Blaber M, Vandell AG (2012) Kallikrein 6 is a novel molecular trigger of reactive astrogliosis. Biol Chem 393:355-367.

Skripuletz T, Hackstette D, Bauer K, Gudi V, Pul R, Voss E, Berger K, Kipp M, Baumgartner W, Stangel M (2013) Astrocytes regulate myelin clearance through recruitment of microglia during cuprizone-induced demyelination. Brain 136:147-167.

Smith KJ, Blakemore WF, McDonald WI (1979) Central remyelination restores secure conduction. Nature 280:395-396.

Stassart RM, Mobius W, Nave KA, Edgar JM (2018) The axon-myelin unit in development and degenerative disease. Front Neurosci 12:467.

Suo Z, Wu M, Ameenuddin S, Anderson HE, Zoloty JE, Citron BA, AndradeGordon P, Festoff BW (2002) Participation of protease-activated receptor-1 in thrombin-induced microglial activation. J Neurochem 80:655-666.

Sweeney AM, Fleming KE, McCauley JP, Rodriguez MF, Martin ET, Sousa
AA, Leapman RD, Scimemi A (2017) PAR1 activation induces rapid changes in glutamate uptake and astrocyte morphology. Sci Rep 7:43606.

Taylor LC, Puranam K, Gilmore W, Ting JP, Matsushima GK (2010) 17beta-estradiol protects male mice from cuprizone-induced demyelination and oligodendrocyte loss. Neurobiol Dis 39:127-137.

Vandell AG, Larson N, Laxmikanthan G, Panos M, Blaber SI, Blaber M, Scarisbrick IA (2008) Protease activated receptor dependent and independent signaling by kallikreins 1 and 6 in CNS neuron and astroglial cell lines. J Neurochem 107:855-870.

Vega-Riquer JM, Mendez-Victoriano G, Morales-Luckie RA, Gonzalez-Perez O (2019) Five decades of cuprizone, an updated model to replicate demyelinating diseases. Curr Neuropharmacol 17:129-141.

VonDran MW, Singh H, Honeywell JZ, Dreyfus CF (2011) Levels of BDNF impact oligodendrocyte lineage cells following a cuprizone lesion. J Neurosci 31:14182-14190.

Wies Mancini VSB, Pasquini JM, Correale JD, Pasquini LA (2019) Microglial modulation through colony-stimulating factor-1 receptor inhibition attenuates demyelination. Glia 67:291-308.

Wong AW, Xiao J, Kemper D, Kilpatrick TJ, Murray SS (2013) Oligodendroglial expression of TrkB independently regulates myelination and progenitor cell proliferation. J Neurosci 33:4947-4957.

Yeung MSY, Djelloul M, Steiner E, Bernard S, Salehpour M, Possnert G, Brundin L, Frisen J (2019) Dynamics of oligodendrocyte generation in multiple sclerosis. Nature 566:538-542.

Yoon H, Radulovic M, Drucker KL, Wu J, Scarisbrick IA (2015) The thrombin receptor is a critical extracellular switch controlling myelination. Glia 63:846-859.

Yoon H, Radulovic M, Walters G, Paulsen AR, Drucker K, Starski P, Wu J, Fairlie DP, Scarisbrick IA (2017) Protease activated receptor 2 controls myelin development, resiliency and repair. Glia 65:2070-2086.

Yoon H, Radulovic M, Scarisbrick IA (2018) Kallikrein-related peptidase 6 orchestrates astrocyte form and function through proteinase activated receptor-dependent mechanisms. Biol Chem 399:1041-1052.

Yun SP, Kam TI, Panicker N, Kim S, Oh Y, Park JS, Kwon SH, Park YJ, Karuppagounder SS, Park H, Kim S, Oh N, Kim NA, Lee S, Brahmachari S, Mao X, Lee JH, Kumar M, An D, Kang SU, et al. (2018) Block of Al astrocyte conversion by microglia is neuroprotective in models of Parkinson's disease. Nat Med 24:931-938.

Zeger M, Popken G, Zhang J, Xuan S, Lu QR, Schwab MH, Nave KA, Rowitch D, D'Ercole AJ, Ye P (2007) Insulin-like growth factor type 1 receptor signaling in the cells of oligodendrocyte lineage is required for normal in vivo oligodendrocyte development and myelination. Glia 55:400-411. 